\title{
A Parametrization, Based on Sea Ice Morphology, of the Neutral Atmospheric Drag Coefficients for Weather Prediction and Climate Models
}

Christof Lüpkes, ${ }^{1}$ Vladimir M. Gryanik, ${ }^{1,2}$ Jörg Hartmann, ${ }^{1}$ and Edgar L Andreas ${ }^{3}$

C. Lüpkes, Alfred Wegener Institute for Polar and Marine Research, Postfach 120161, D-27515

Bremerhaven, Germany. (Christof.Luepkes@awi.de)

${ }^{1}$ Alfred Wegener Institute for Polar and

Marine Research, Bremerhaven, Germany

${ }^{2}$ A.M. Obukhov Institute of Atmospheric

Physics, Russian Academy of Sciences,

Moscow, Russia

${ }^{3}$ NorthWest Research Associates, Inc.

Lebanon, New Hampshire 03766, USA 
Abstract. A hierarchy of parametrizations of the neutral $10 \mathrm{~m}$ drag coefficients over polar sea ice with different morphology regimes is derived on the basis of a partitioning concept that splits the total surface drag into contributions of skin drag and form drag. The new derivation, which provides drag coefficients as a function of sea ice concentration and characteristic length scales of roughness elements, needs fewer assumptions than previous similar approaches. It is shown that form drag variability can explain the variability of surface drag in the marginal sea ice zone (MIZ) and in the summertime inner Arctic regions. In the MIZ, form drag is generated by floe edges; in the inner Arctic, by edges at melt ponds and leads due to the elevation of the ice surface relative to the open water surface. It is shown that an earlier fit of observed neutral drag coefficients is obtained as a special case within the new concept when specific simplifications are made which concern the floe and melt pond geometry. Due to the different surface morphologies in the MIZ and summertime Arctic, different functional dependencies of the drag coefficients on the sea ice concentration result. These differences cause only minor differences between the MIZ and summertime drag coefficients in average conditions, but they might be locally important for atmospheric momentum transport to sea ice. The new parametrization formulae can be used for present conditions but also for future climate scenarios with changing sea ice conditions. 


\section{Introduction}

Sea ice consists in the polar marginal sea ice zones (MIZ) of floes with diameters between about one meter and one kilometer. The floes are surrounded by open sea water which sometimes contains also slush and brash ice. In such regions, the surface roughness varies strongly on a scale of tens or hundreds of meters depending, amongst others, on the size of floes and distance between floes. However, in most state-of-the-art climate and sea ice models (e.g., ECHAM: Roeckner et al., 2003; FESOM: Timmermann et al., 2009]), the variability of sea ice surface roughness is not taken into account and surface roughness does not depend on sea ice characteristics.

A study of Zhang and Rothrock [2003] gives a hint as to the possible progress which could be expected by a more detailed parametrization of surface drag. In their coupled global ice-ocean model, they prescribe one constant value for the atmospheric drag coefficient which is based on a number of values for different ice types proposed by Overland [1985]. Results show that the sea ice drift depends strongly on the chosen value, and much better agreement with observations was possible with an optimized drag coefficient.

Nowadays, there is in several models (e.g., in the regional WRF model [Skamarock et al., 2008] and also in the climate model COSMO-CLM (http://www.clm-community.eu) the possibility to account for fractional sea ice cover with different roughness lengths for ice and open water. Fluxes are obtained as an average over both surface types. This results in a linear dependence of the neutral drag coefficient on the sea ice concentration, as explained in Birnbaum and Lüpkes [2002]. As will be shown later, this linear dependence does not hold in a more detailed treatment of surface drag. 
The parametrization of the MIZ surface roughness has been discussed intensively during the last three decades, starting with studies by Overland [1985] and Guest and Davidson [1987]. A general finding of Andreas et al. (1984), Hanssen-Bauer and Gjessing [1988], Stössel and Claussen [1993], Mai et al. [1996], Birnbaum and Lüpkes [2002], Lüpkes and Birnbaum [2005], and Lüpkes et al. [2012] was that atmospheric momentum fluxes are influenced not only by the skin drag of the open water surface and of the more or less plane ice floe surfaces but also by the form drag caused by the edges of floes, where often small ridges form due to floe collisions. So, the effective atmospheric drag over the MIZ was parametrized by accounting for both skin drag and form drag using schemes of different complexity. The inclusion of the form drag results in a nonlinear dependence of the drag coefficient on sea ice concentration. Recently, Lu et al. [2011] used an analogous concept to parametrize the ice-ocean drag coefficient.

The parametrizations of atmospheric surface drag over the MIZ which have been developed for the scale of regional climate and weather prediction models reproduce drag coefficients observed, for example, by Andreas et al. [1984], Guest and Davidson [1987], and Fairall and Markson [1987] fairly well, as shown by Lüpkes and Birnbaum [2005]. But these parametrizations still cannot explain the complete range of scatter of observed drag coefficients for a given sea ice concentration.

Certainly, marginal sea ice zones are small compared with the whole region covered by the polar oceans so that the relevance of drag parametrizations over the MIZ seemed to be limited. However, recently Andreas et al. [2010] (abbreviated by AN10) have demonstrated on the basis of data from the SHEBA campaign [Uttal et al., 2002] that the drag over a melt-pond-covered sea ice surface, which is typical during summer for 
the whole Arctic, is also influenced by form drag. Here, the form drag is caused by the elevation of ice above the melt pond surface or by the floe edges in case of leads.

Considering the evolution of drag coefficients measured at the SHEBA ice station during summer 1998, the form drag effect is obvious since drag increases with decreasing sea ice cover (AN10). At an ice cover of about $50 \%$, it attains a maximum value. A maximum at 50-60\% ice cover is also found by Mai et al. [1996] for the marginal ice zone; they explain this as an effect of the form drag caused by an increasing number of floe edges with increasing sea ice concentration $A$ and with a sheltering of the atmospheric flow downstream of floes for $A$ larger than about 50-60\%.

Thus, the finding of AN10 represents an important step towards a more general parametrization of air-ice exchange in ice-covered regions since it shows that the drag parametrization concept originally derived for the small marginal ice zones with specific sea ice morphology can be used for a much larger geographical region. AN10 fitted the available MIZ surface drag data and SHEBA data by a second order polynomial and proposed to use this parametrization in the MIZ and inner summer Arctic. A drawback is that the proposed formula does not allow accounting for specific conditions in certain regions and represents only average conditions. A similar strategy was chosen recently by Weiss et al. [2011], who proposed an average roughness length for the Weddell Sea region.

The main goal of the present work is, however, to derive a parametrization which allows more flexibility than the AN10 formula in adopting the parametrization to varying conditions over the Arctic Ocean while being as simple as the AN10 fit. It is also shown that the latter can be derived similarly as the Lüpkes and Birnbaum [2005] detailed model 
by applying several modifications (e.g., no blending height, simpler floe topography). The new scheme, which will be based again on the concept of drag partitioning, is then similar to that proposed by Hanssen-Bauer and Gjessing [1988] but differs in several aspects as, for example, in its functional dependence of the drag coefficients on sea ice parameters.

The concept of drag partitioning due to which the total drag of a surface can be separated into contributions by skin drag and form drag is artificial and goes back to Schlichting (1936). The partitioning concept is often used for surfaces where there is a clear scale separation between large roughness elements distributed irregularly in space and elements which are much smaller and more or less regularly distributed with much smaller distances between each other [e.g., Andreas, 1995]. Raupach [1992] as well as Shao and Yang [2008], for example, review the different approaches possible to treat the effect of the larger roughness elements. In effect, the concept of drag partitioning allows a simple way to derive a functional dependence of the drag force on the geometry and area coverage of large roughness elements, which are represented in our case by floes.

It is the goal of the present approach to treat the form drag finally as a modification of the neutral drag coefficient which can be reformulated in terms of a roughness length concept using surface layer theory. Thereby, the main difficulty in deriving the drag coefficients over the MIZ and summer sea ice consists in an adequate description of the effect of different sea ice morphologies.

For moderate sea ice concentrations, floes in the MIZ can be assumed as drifting without direct contact with each other. The surface morphology in the inner melting Arctic is at least, in the first stage, complementary to the MIZ with individual ponds surrounded 
by connected sea ice (Figure 6). As will be elaborated later, this change in the sea ice morphology causes a change in the derivation of the drag coefficients.

\section{Motivation and Goals}

AN10 based their parametrization of the effective 10 -m neutral drag coefficient $C_{d n 10}$ on observations. As mentioned above, they focused on two different kinds of data. The first ones are related to the summer months of the SHEBA campaign carried out in the central Beaufort Gyre, when the open water fraction was due to leads and melt ponds. The second type of data set was obtained over the Arctic and Antarctic MIZ during observations from ship and aircraft in various seasons. Data are available for the Antarctic from Andreas et al. [1984], and for the Arctic from the campaign MIZEX [Guest and Davidson, 1987; Anderson, 1987] and Birnbaum and Lüpkes [2002], who compiled results from the campaigns REFLEX described in Mai et al. [1996], Kottmeier et al. [1994], and Hartmann et al. [1992, 1994].

AN10 fitted the $C_{d n 10}$ observations with a second-order polynomial

$$
10^{3} C_{d n 10}=1.500+2.233 A-2.233 A^{2}
$$

where $A$ is the sea ice concentration. They compared the results following from this equation with drag coefficients obtained for particular conditions from a parametrization by Lüpkes and Birnbaum [2005] (in the following abbreviated by LB05), who applied the concept of drag partitioning based on the same splitting concept as proposed by Shao and Yang [2008] for surfaces with large roughness elements in a smooth environment. It accounts for the form drag by obstacles (ice floes) and for skin drag caused by both the obstacles and the smooth surface (water). 
Thus the drag coefficient in LB05 is based on the formulation

$$
C_{d n 10}=(1-A) C_{d, w}+A C_{d, i}+C_{d, f}
$$

$C_{d, f}$ is the form drag coefficient accounting for the additional drag caused by the edges of floes. $C_{d, i}$ and $C_{d, w}$ are the skin drag coefficients over sea ice and over open water. LB05 proposed to use a fit of $C_{d, f}$ to results of their more complex parameterization, which is given as

$$
C_{d, f}=0.34 \cdot 10^{-3} A^{2} \frac{(1-A)^{0.8}+0.5(1-0.5 A)^{2}}{\alpha_{r}+90 A}
$$

$a_{r}$ is the aspect ratio $D_{i} / h_{f}$ (mean floe length/mean freeboard of floes), where

$$
D_{i}=h_{f} \frac{31}{(1-A)}
$$

and hence

$$
\alpha_{r}=\frac{D_{i}}{h_{f}}=\frac{31}{(1-A)} .
$$

The above equations with the dimensionless factor 31 arise from a fit to aircraft observations carried out by Mai (1995).

AN10 inserted $C_{d, i}=1.5 \cdot 10^{-3}, C_{d, w}=1.4 \cdot 10^{-3}$ in equation (2) as well as equation (5) in (3) and obtained then from equation (3) results which differ only slightly from their polynomial fit given by equation (1) (Figure 3 in AN10).

The question arises, why results are so similar from both formulae. To give a first answer, we show in Appendix B that equation (1) can be reformulated after some algebra following the basic concept given by equation (2) in terms of the second-order polynomial

$$
C_{d n 10}=(1-A) C_{d, w}+A C_{d, i}+4 C_{d, f \max } A(1-A)
$$


where the last term is the form drag contribution and $C_{d, f \max }$ is the maximum value of the form drag coefficient. Choosing $C_{d, f \max }=0.558 \cdot 10^{-3}$ and the skin drag coefficients as mentioned before, the results of (6) are the same as those obtained by the AN10 equation (1). For simplicity, we will call equation (6) in the following the AN10 equivalent $C_{d}$.

Comparing furthermore equations (6) and (1), it follows that the form drag coefficient in the AN10 equation is given by

$$
C_{d f}=2.23 \cdot 10^{-3} A(1-A)
$$

This can be compared with the form drag coefficient (3) in the LB05 parametrization, which we can rewrite as

$$
C_{d f}=10.54 \cdot 10^{-3} A(1-A) F(A)
$$

with

$$
F(A)=A \frac{(1-A)^{0.8}+0.5(1-0.5 A)^{2}}{1+3 A(1-A)}
$$

From Figure 6 it becomes clear that $F$ is a smooth function, and it can be roughly approximated by a constant so that the form of equations (7) and (8) becomes the same. With $F=0.21$, results from equation (8) are quite similar (for $A>0.15$ ) to those using (9). We will see in the next section that the $F$ function represents the effect of sheltering of the flow by floe edges and ridges and the effect of dynamic pressure on the edges. The term $A(1-A)$ represents only the effect of the floe distribution affecting the number density of floe edges.

Both parametrization equations (1) and (2) with (3) have drawbacks. Although the AN10 fit is based on a large set of observed data and thus represents the mean conditions, there is no possibility to take into account extraordinary conditions with, for example, 
rougher or smoother ice which might occur due to the variability of aspect ratio $\alpha_{r}$ and skin drag. The shortcoming of the LB05 curve is that it represents a fit only of a physical model of LB05.

Based on these findings, three goals are formulated which are addressed in the following section. The first goal is to derive, from physical principles, a parametrization of the neutral drag coefficient which is as easy to apply to models as the fitting equations (1) and (2) with (3). Thereby, the present work concentrates on the marginal sea ice zone (Section 3) and on the inner summertime Arctic (Section 4), where we account for the impact of floe edges (with ridges at these edges) and of edges at melt ponds and leads. In the region of the Fram Strait MIZ, the impact of large ridges is mostly small, as found by Mai et al. (1996); however, in the inner polar sea ice regions, the impact of ridges can be large [Garbrecht et al., 2002]. This impact is, however, not the focus of the present work.

The second goal is to investigate to what extent the differences in the sea ice morphology during summer and in the MIZ affect the drag parametrization. The third goal is to identify the ranges of applicability of the AN10-type and LB05-type parametrizations.

Parametrizations for the MIZ and inner Arctic are derived in Sections 2 and 3. After a discussion of their region of applicability, practical recommendations concerning the use of these parametrizations are given in Section 5 .

\section{Physical Derivation of $C_{d}$ over Fractional Sea Ice}

\subsection{Surface Drag Over the Marginal Sea Ice Zone}

The most detailed model in LB05 represents a further development of the approach of Hanssen-Bauer and Gjessing [1988] (HBG88), who calculated the form drag caused by the edges of floes under the assumption of a periodic distribution of floes of length 
$D_{i}$ and distance $D_{w}$ to each other. HBG88 calculated form drag based on the dynamic pressure on the floe edges using a logarithmic wind profile modified by a simple function accounting for the sheltering effect of floes. The main change introduced by LB05 consisted in the generalization of the HBG88 model with 2D-floes represented by their length and freeboard to a model with 3D-quadratic floes of edge length $D_{i}$ and freeboard $h_{f}$. The effect of this assumption is discussed in Sections 3.1.3 and 4.

Furthermore, LB05 combined the form drag concept with a flux aggregation scheme of Claussen [1990] (see also Grötzner et al. [1996]), including the determination of a surface layer blending height. Another difference was that a pancake-like floe structure was assumed with small ridges at the floe edges as a result of floe collisions.

A disadvantage of this model was that the resulting implicit formulae for the surface drag coefficients were too complex to be used as a basis for the analytical derivation of simple parametrizations so that equation (2) with (3) represented only a fit to the results of the complex scheme rather than a derivation of the functional dependence of $C_{d n 10}$ on $A$. It will be shown in the following that a parametrization model which is closer to the original HBG88 model will allow the derivation of an explicit analytical expression for $C_{d n 10}$ which contains furthermore the AN10 equivalent $C_{d}$ under certain simplifying assumptions.

The goal of the following detailed derivation is to clarify the physical aspects behind our parametrization.

\subsubsection{Derivation of an analytical expression for form drag}


As LB05, we assume 3D floes with freeboard $h_{f}$, where the latter can be influenced by the average ridge height on the floe (e.g., at its upstream edge). The floes can have different shapes, but we assume that the area of a single floe is

$$
S_{i}=c_{s} D_{i}^{2}
$$

Here, $D_{i}$ is the effective edge length for form drag (basically, the cross-wind dimension of the floe), and $c_{s}$ is a shape factor describing the deviation of the mean floe shape from a square (e.g., $c_{s}=1$ for squares; $c_{s}=\pi / 4$ for circles).

A domain is now considered with area $S_{t}>>S_{i}$ containing $N$ identical floes so that

$$
A=c_{s} \frac{N D_{i}^{2}}{S_{t}}
$$

We stress that at this stage no assumption is necessary about the distribution of floes, including their distances to each other. $S_{t}$ could represent, for example, a model's grid cell area or the MIZ domain. The effective neutral $10 \mathrm{~m}$ drag coefficient is then given by equation (2) with the form drag coefficient

$$
C_{d f}=\frac{\tau_{d}}{\rho U_{10}^{2}}
$$

where $\rho$ is the air density, $U_{10}$ is the 10 -m wind speed, and $\tau_{d}$ is the momentum flux per unit area of the domain. We derive $\tau_{d}$ in two steps.

As a first step, we determine the mean dynamic pressure $P_{d}$ on the floe edges as the force $f_{d}$ per unit of frontal upstream area of the $N$ floe edges with freeboard $h_{f}$ and length $D_{i}$ in the domain $S_{t}$ as

$$
P_{d}=\frac{f_{d}}{N h_{f} D_{i}}
$$


with $f_{d}$ defined by the integral

$$
f_{d}=N c_{w} S_{c}^{2} \int_{0}^{D_{i}} \int_{z_{0, w}}^{h_{f}} \frac{\rho U^{2}}{2} d z d y
$$

The factor $N$ appears due to the summation over all $N$ floes. $U$ is the height dependent upstream wind speed, which does not contain sheltering of the flow. The latter is considered as independent of $U$ and $z$ and is accounted for by the sheltering function $S_{c}$ such that $S_{c} \rightarrow 1$ for large distances between floes and $S_{c} \rightarrow 0$ for small distances.

The height integration is from the aerodynamic roughness over open water, $z_{0, w}$ to $h_{f}$. $c_{w}$ is the coefficient of resistance of an individual floe. We define $c_{w}=f_{d} / f_{d, \infty}$, where

$$
f_{d, \infty}=\int_{0}^{D_{f}} \int_{z_{0, w}}^{h_{f}} p d z d y
$$

with the dynamic pressure $p=\rho U^{2} / 2$. Thus $f_{d, \infty}$ is the force of the unsheltered flow on a frontal area $S_{f}=D_{f}\left(h_{f}-z_{0, w}\right)$ of a vertical wall.

Now, as the second step, we apply the assumption

$$
P_{d} N D_{i}\left(h_{f}-z_{0, w}\right)=\tau_{d} S_{t}
$$

to relate the force which the flow acts on the $N$ floes to the drag force acting on the surface of domain $S_{t}$. Solving this equation for $\tau_{d}$ and assumimg $z_{0, w}<<h_{f}$, we obtain the surface drag as

$$
\tau_{d}=P_{d} \frac{N h_{f} D_{i}}{S_{t}}=\frac{1}{c_{s}} P_{d} \frac{h_{f}}{D_{i}} A
$$

where (11) was used for $S_{t}$.

With (17) and (12), the MIZ drag coefficient is then given by

$$
C_{d n 10}=(1-A) C_{d, w}+A C_{d, i}+P \frac{h_{f}}{D_{i}} A
$$


where $P=P_{d} /\left(c_{s} \rho U_{10}^{2}\right)$ is proportional to the dynamic pressure contribution of floe edges to the form drag coefficient. We insert (13) with (14), and after assuming a logarithmic wind profile $U=\left(u_{*} / \kappa\right) \ln z / z_{0}$, the integrals in equation (14) can be solved (see HBG88). This results in

$$
P=\frac{c_{e}}{2}\left[\left[\ln \left(\frac{h_{f}}{z_{0, w}}\right)-1\right]^{2}+1-2 \frac{z_{0, w}}{h_{f}}\right] S_{c}^{2}\left[\ln \left(\frac{10}{z_{0, w}}\right)\right]^{-2}
$$

with the effective resistance coefficient

$$
c_{e}=\frac{c_{w}}{c_{s}}
$$

$c_{e}$ depends on both the aerodynamic resistance coefficient of individual floes $c_{w}$ and the shape factor $c_{s}$ (equation 10).

For typical values of $h_{f}$ and $z_{0, w}$, a very good approximation of this equation with a deviation smaller than $1 \%$ of $P$ is

$$
P=\frac{c_{e}}{2}\left[\frac{\ln \left(h_{f} / z_{0, w}\right)}{\ln \left(10 / z_{0, w}\right)}\right]^{2} S_{c}^{2}=P_{0} S_{c}^{2}
$$

So, we obtain finally

$$
C_{d n 10}=(1-A) C_{d, w}+A C_{d, i}+\frac{c_{e}}{2}\left[\frac{\ln \left(h_{f} / z_{0, w}\right)}{\ln \left(10 / z_{0, w}\right)}\right]^{2} S_{c}^{2} \frac{h_{f}}{D_{i}} A
$$

Note that although a similar equation for $P$ can be found in the HBG88 derivation, which is used for the parametrization of form drag, they propose no drag coefficient.

$c_{w}$ is available from measurements where the resistance is mainly caused by ridges formed at the floe edges due to floe collisions. Such $c_{w}$ measurements can be taken from Banke and Smith [1975] (reanalyzed by Garbrecht et al. [1999, 2002]). Results imply that the allowed range is large, with $c_{w}$ between 0.1 and 0.5 .

We can only speculate about the shape parameter $c_{s}$. In case of a circle, $c_{s}=\pi / 4 \approx 0.8$; for squares, $c_{s}=1$ but also larger values than 1 are possible. In nature, there are D R A F T 
sometimes situations with elongated floe structures. For example, for ellipses, $c_{s}$ will depend on the ratio of the axes. In this case, the form drag would depend even on the wind direction; however, in present models, there is no possibility to predict floe orientation. The effect of average wind direction could be included by multiplying $c_{e}$ by a factor describing the effect of averaging over the wind direction.

Considering these uncertainties, we treat the fraction $c_{e}=c_{w} / c_{s}$ as a tuning parameter. Our results for $C_{d n 10}$, explained in the next subsections, compare well with observations carried out for various wind directions relative to the floe orientation when $c_{e}=c_{w} / c_{s}=0.3 \pm 0.1$

It will be shown below that $S_{c}$ can be approximated by a formula depending only on A. An application of equation (22) in a climate or weather prediction model is thus possible, provided that $A, h_{f}$, and $D_{i}$ are known. In the best case, the model consists of a coupled atmosphere and sea ice model. Then, $A$ and $h_{f}$ (which is related to the sea ice thickness) are available from prognostic equations. It is also possible to introduce a prognostic equation for $D_{i}$ (see Birnbaum [1998]); but, usually, $D_{i}$ is not predicted and has to be parametrized.

In the case that there is no information on $h_{f}$ and $D_{i}$ from a model, we now have two possibilities. The first is to claim simply that equation (22) should give the same results as the AN10 equivalent $C_{d}$ (equation 6) using the argument that the results agree on average fairly well with measured drag coefficients. This would imply the use of an aspect ratio $D_{i} / h_{f}$ being proportional to $[1 /(1-A)]$. Assuming, furthermore, a constant freeboard and roughness length $z_{0, w}$ in the pressure term $P$, equation (22) and the AN10 
equivalent $C_{d}$ have the same form. Note, that by this method no further assumptions on floe characteristics than already made are necessary.

However, the above method ignores possible deviations from the average aspect ratio and the existence of different functional dependencies. So, the second possibility is to parametrize $h_{f}$ and $D_{i}$, preferably as a function of $A$ while keeping in mind that both variables are influenced also by other quantities, such as the wave height, temperature, and wind speed conditions during the floe's life time. Also, the geographical position plays a role since freeboard is closely connected with the sea ice thickness, which is not uniform over the Arctic. One can expect that, in certain regions and under certain flow conditions, the dependence on $A$ will remain after averaging the data for given $A$ and hence removing all other dependencies. As discussed in LB05, the eastern Fram Strait is one such region, at least for off-ice flow.

\subsubsection{Parametrization of floe length and freeboard}

We reconsidered the Fram Strait aircraft data of Hartmann et al. [1992] and Kottmeier et al. [1994] for $h_{f}$ and $D_{i}$, which they obtained from 10-km flight sections (Figure 6). The scatter in the $h_{f}$ and $D_{i}$ data for a given $A$ hint to the dependence on other variables besides $A$. Another reason for the scatter consists in the measurement errors, for example, of $D_{i}$. As explained in more detail by Hartmann et al. (1994), $D_{i}$ is not directly measured but derived from the measured edge length of floes in a domain of $10 \mathrm{~km}$ length and $60 \mathrm{~m}$ width. This process results in a mean error of roughly $\pm 30 \%$, as derived by Mai [1995] (shown by error bars in Figure 6). Measurements of $h_{f}$ are more accurate, with an uncertainty of $\pm 20 \%$. 
Despite the large uncertainties in the data (Figure 6), it seems at least justified to assume that $h_{f}$ increases with increasing $A$. A possible physical explanation for this dependence is that, during off-ice drift situations as being typical for cold-air outbreaks with a widespread MIZ, $A$ decreases toward the ice edge. This is simply due to the dispersion of floes caused by wind and ocean current. The decrease of freeboard toward the ice edge is then caused by the melting of floes along their drift trajectories in the opening water.

To study the implications of a specific choice for the functional dependence on $A$, we consider three possible parametrizations $h_{f}(A)$, whose results are all within the range of measured freeboard. They are given by (see also Figure 6)

$$
\begin{aligned}
& h_{f}(A)=h_{\max }[1-\exp (-5.9 A)] \\
& h_{f}(A)=h_{\max } \sqrt{A} \\
& h_{f}(A)=h_{\max } A+h_{\min }(1-A) .
\end{aligned}
$$

Equation (23), with $h_{\max }=0.49 \mathrm{~m}$ is equivalent to the fit proposed by Mai [1995] and used by LB05; but - as can be seen from Figure 6 - the variability is large and also other fits seem to be reasonable, like that by equation (24).

The idea behind (23) and (24) was to obtain $h_{f} \rightarrow 0$ for $A \rightarrow 0$. However, this behavior near $A=0$ is not clearly seen from the data, and there are also arguments for the existence of a minimum floe size larger than 0 . One is that floes melt faster from their bottoms than from the sides. For this reason and since all further results obtained from the different approaches differed only slightly from those using a simple linear fit, we decided to use equation (25) as the basic $h_{f}$ approximation. The possible ranges are [0.08 $\left.\mathrm{m}, 0.4 \mathrm{~m}\right]$ for the minimum freeboard $h_{\min }$ and $[0.35 \mathrm{~m}, 0.65 \mathrm{~m}]$ for the maximum freeboard $h_{\max }$. In 
the following, the values $h_{\min }=0.286 \mathrm{~m}$ and $h_{\max }=0.534 \mathrm{~m}$ are always used based on a least square fit.

Considering $D_{i}$ in Figure 6, a reasonable approximation is given by

$$
D_{i}=D_{\min }\left(\frac{A_{\star}}{A_{\star}-A}\right)^{\beta},
$$

where $A_{\star}$ is introduced instead of the value 1 to avoid a singularity at $A=1 . A_{\star}$ is related to the smallest floe length $D_{\min }$ for $A \rightarrow 0$ and the largest one $D_{\max }$ for $A \rightarrow 1$ by

$$
A_{\star}=\frac{1}{1-\left(D_{\min } / D_{\max }\right)^{1 / \beta}}
$$

According to Figure 6, values for $D_{\min }$ can vary between about $2 \mathrm{~m}$ and $25 \mathrm{~m}$. Since $D_{\min }$ is usually much smaller than $D_{\max }$, the value of $A_{\star}$ is close to 1 . We used here $D_{\text {min }}=8 \mathrm{~m}$, which results in the best agreement of the calculated drag coefficients with Fram Strait observations (see below).

The impact of $D_{\max }$ on drag coefficients turned out to be small. We chose in Figure 6 and later a value of $300 \mathrm{~m}$, but results are similar for $D_{\max }$ in the range between $200 \mathrm{~m}$ and $1000 \mathrm{~m}$.

The $D_{i}$ curve depends also on the exponent $\beta$. Reasonable $\beta$ values in the sense that they result in drag coefficients that have been observed are between 0.2 and 1.8 .

The calculation of $A_{\star}$ with the above equation is the most solid way; but in practice, to save cpu time, it might be of advantage to use $A_{\star}=1$ despite the mentioned singularity. As will be shown in section 3.1.4, one should be aware in this case also of unphysical floe number densities and of the later derived distances between floes for $A \rightarrow 1$.

Despite the uncertainties in the observations of $D_{i}$ and $h_{f}$, they are accurate enough to exclude certain parametrizations. As an example, Figure 6 contains a curve represent- 
ing the assumption of a constant $D_{i}(25 \mathrm{~m})$. It is obvious that such a parametrization combined with the linear fit to $h_{f}$ contradicts the aspect ratios $\alpha_{r}$ derived from the observations. An alternative method would have been to parametrize $\alpha_{r}$ directly. However, it is useful to have separate equations for $D_{i}$ and $h_{f}$ since $h_{f}$ could also be obtained from sea ice models; for such models, only $D_{i}$ is required (see section 5 ) in the parametrization of the drag coefficient.

\subsubsection{Parametrization of sheltering and dynamic pressure}

As a further step towards the final suggestion for the MIZ drag parametrization, we investigate the behavior of the pressure and sheltering terms $P$ and $S_{c}$ as a function of $A$. A simple $S_{c}$ parametrization is given in HBG88 by

$$
S_{c}=\left(1-\exp \left(-s D_{w} / h_{f}\right)\right)
$$

with the dimensionless constant $s$ and the distance between floes $D_{w}$. Comparing results from equation (28) with results obtained by wind tunnel observations and modeling (see e.g., Lopez et al. [2005]) and interpreting $D_{w}$ as the distance to an obstacle, it is obvious that the value for $s$ proposed by HBG88 $(s=0.18)$ results in a much too large sheltering effect. Better agreement is obtained with $s=0.5$.

The application of equation (28) in the present drag parametrization requires the specification of $D_{w}$ as the distance between floes and, hence, an assumption on their distribution. One possibility among many others was proposed by LB05, who assumed periodically distributed quadratic floes, which results in

$$
D_{w}=D_{i}(1-\sqrt{A}) / \sqrt{A} .
$$


Inserting this equation in (28); using (26) with $D_{\min }=8 \mathrm{~m}, D_{\max }=300 \mathrm{~m}$, and $\beta=1$; as well as (25), we obtain the results in Figure 6. This shows that the sheltering reduces form drag only for very large ice concentration, where according to Figure 6 (see also next subsection) the distance between floes is less than 4-5 m, obviously a critical value for the beginning of sheltering. So, for $A<0.97$, the decrease of form $\operatorname{drag}$ with increasing $A$ is solely a consequence of the floe geometry dependence on $A$, which will occur in the $C_{d}$ formulae as the product $A\left(A_{\star}-A\right)$ (see below, equation $\left.(33)\right)$.

Finally, since the sheltering has only small impact, we can approximate $S_{c}$ either by

$$
S_{c}^{2}=1-\exp \left[-s_{l} \beta(1-A)\right]
$$

with $s_{l}=22$ and $\beta$ as in equation (26), or even more simply by the power law

$$
S_{c}^{2}=(1-A)^{1 /(10 \beta)}
$$

Since an increase of $\beta$ is equivalent to larger floe sizes, the above formula takes into account the effect that distances between floes increase and thus sheltering decreases when the same $A$ is realized by larger floes.

A comparison of results between the three parametrizations (28), (30), and (31) is shown in Figure 6. Results of equations (28) and (30) differ only slightly from each other; while, in comparison with results from equation (28), the function (31) overestimates $S_{c}$ for $A>0.8$ and underestimates $S_{c}$ for $A<0.8$, respectively. However, for $\beta \geq 1$, the form drag influence is never larger than about $40 \%$ of total drag so that the rough parametrization (31) is allowed. We found that for the Fram Strait conditions as observed during REFLEX even $S_{c}=1$ can be used (Figure 6 ). 
Also the dynamic pressure term $P_{0}$ (equation (21)) varies only little with $A$ (Figure 6) so that the $A$ dependence could be neglected in its parametrization by assuming a constant $h_{f}$, as we do later in the most simplified version of the $C_{d}$ parametrization.

\subsubsection{Effect of $D_{i}$ on floe numbers and on $D_{w}$}

Although not needed for the derivation of drag coefficients, we can check the plausibility of our previous assumptions on floe geometry by calculating the number density $n$ of floes as a function of $A$. We obtain from equation (11)

$$
n=\frac{N}{S_{t}}=c_{s} \frac{A}{D_{i}^{2}}
$$

Figure 6 shows that the influence of the $D_{i}$ parametrization (equation 26or 4 ) is large for both $n$ and $D_{w}$. It is obvious that for $A \rightarrow 1$ only those $D_{w}$ values obtained with equations (26) and (27) are physically realistic since for $A=1$ the distance between floes has to become zero. For $\beta \geq 1$, the approximation $A_{\star}=1$ causes unphysical values at high ice concentrations.

The floe numbers are less sensitive to the choice of $A_{\star}$ (small differences between dashed and solid lines in Figure 6). There is, however, a large difference between the results for $n$ when we use equation (26) instead of (4). This holds especially for the behavior at $A \rightarrow 0$. The Mai [1995] parametrization (equation 4) produces a strongly increasing number of floes for $A \rightarrow 0$ while the distance between floes decreases. One cannot rule out this behavior in nature, but a monotonic increase of $D_{w}$ with increasing $A$ and $n$ going to zero seems to be more plausible. So, the validity of the LB05 drag parametrization using the Mai [1995] formulae is limited roughly to the region with $0.1<A<0.99$; and we prefer to use equation (26) for $D_{i}$ in the following since it has fewer limitations. 
In the Fram Strait cases shown in Figure 6, $A$ was mostly decreasing in the MIZ with distance $y$ from the compact ice. For $0.4<A<1$, this means that both $n$ and $D_{i}$ decrease likewise with $y$ (Figures 6 and 6), which is a finding confirmed by Inoue et al. [2004] by observations during flights over sea ice in the Sea of Okhotsk.

\subsection{5. $\mathrm{C}_{\mathrm{dn10}}$ of the MIZ using different approximations}

Using now the linear fit $h_{f}=h_{f l}=h_{\max } A+h_{\min }(1-A)$ given by equation $(25)$ and for $D_{i}$ equation (26), we obtain from equation (22)

$$
C_{d n 10}=(1-A) C_{d, w}+A C_{d, i}+C_{f}\left(A_{\star}-A\right)^{\beta} A
$$

with

$$
C_{f}=\frac{c_{e}}{2}\left[\frac{\ln \left(h_{f l} / z_{0, w}\right)}{\ln \left(10 / z_{0, w}\right)}\right]^{2} S_{c}^{2} \frac{h_{f l}}{D_{\min } A_{\star}^{\beta}} .
$$

$S_{c}^{2}$ can be determined by equation (30) or more simply by (31), if $\beta \geq 1$ is chosen. The value of $\beta$ can depend on specific conditions like ridging in different ice regimes. This is discussed later.

Before we examine this parametrization by comparing its results for $C_{d n 10}$ with data, we show that with further simplifications, ignoring partly the strict physical dependencies, the AN10-equivalent parametrization results from the above form.

This is done in two steps. The first is to use $S_{c}=1$ and $A_{\star}=1$. The latter assumption leads to the described drawbacks in $D_{w}$ and $n$. On the other hand, with $S_{c}=1$ or by using parametrizations (30) or (31) instead of (28), these quantities no longer occur in the formulae for the drag coefficients. Furthermore, the multiplication with $(1-A)$ instead of $A_{\star}-A$ can be seen as a replacement for the neglected sheltering function $S_{c}$ in the above 
formula since herewith form drag reduces to zero for $A=1$, which is in the strict sense the result of sheltering.

The second step is to use a constant value for $z_{0, w}$ and to replace $h_{f l}$ by a constant value $h_{f c}$. This results in

$$
C_{d n 10}=(1-A) C_{d, w}+A C_{d, i}+C_{f}(1-A)^{\beta} A
$$

with

$$
C_{f}=\frac{c_{e}}{2}\left[\frac{\ln \left(h_{f c} / z_{0, w}\right)}{\ln \left(10 / z_{0, w}\right)}\right]^{2} \frac{h_{f c}}{D_{\min }} .
$$

Results of equations $(33,34)$ and $(35,36)$ are shown in Figure 6 for $z_{0, w}=3.27 \cdot 10^{-4} \mathrm{~m}$, which corresponds under neutral conditions to $C_{d, w}=1.5 \cdot 10^{-3}$, the value proposed by AN10. With $c_{e}=0.3, D_{\min }=8 \mathrm{~m}, \beta=1$, and $h_{f c}=0.28 \mathrm{~m}$, we obtain $C_{f}=2.24 \cdot 10^{-3}$. With this value, equations $(35,36)$ and the AN10 equivalent parametrization (6) are nearly identical. However, the best agreement between equations $(33,34)$ and $(35,36)$ is obtained with $h_{f c}=0.41 \mathrm{~m}$, which is the mean value of the REFLEX Fram Strait data. The latter value results in $C_{f}=3.67 \cdot 10^{-3}$.

With $\beta=1$, equation (35) can be rewritten in the notation of AN10 as

$$
C_{d n 10}=C_{d, w}+A\left(C_{d, i}-C_{d, w}+C_{f}\right)-C_{f} A^{2}
$$

Comparison with equation (B4) reveals that in this case $C_{f} / 4$ is the maximum possible form drag.

The results in Figure 6 illustrate that a change of $\beta$ shifts the $C_{d}$ maximum towards larger floe lengths (large $\beta$ ) or smaller floe lengths (small $\beta$ ). Obviously, a change of only $\beta$ can explain a large part of the variability in the observed drag coefficients in different ice edge zones. So, the results with $\beta=0.3$ represent well conditions observed in the Antarctic 
and in the western part of the Fram Strait during MIZEX, while the conditions with free drift as in the eastern part of the Fram Strait (see also LB05) are better reproduced using $\beta=1.4$. So, the new parametrization can partly explain the highest observed values of form drag which was not possible with the LB05 formulae. LB05 obtained the largest values only as an effect of strongly increased skin drag over ice.

We derived now different equations for the MIZ drag coefficients which differ by the degree of approximations. Before we give further hints in Section 5 for practical use, we consider the derivation of the drag coefficient for sea ice conditions in the inner Arctic during the melt season.

\subsection{Drag Coefficients Over Summer Sea Ice in the Inner Arctic}

\subsubsection{Sea ice morphology and melt pond distribution}

In the previous sections, the derivation of the drag parametrization referred to typical MIZ conditions with sea ice occurring as distinct floes. In the inner Arctic, a floe structure is not dominant. Even so, as pointed out by AN10, during summer, form drag may still dominate the drag as in the MIZ because the sea ice surface is elevated relative to the surface of melt ponds and leads. However, as shown by Figure 6, the morphological structure of a sea ice surface covered by melt ponds and leads differs from a surface with fractional sea ice as in the MIZ. There, floes are on average not in direct contact, at least for moderate sea ice concentrations; therefore, individual floes can be well distinguished, while the open water patches are connected. For very high sea ice concentration and in case of melt ponds, the situation is opposite, with disconnected countable ponds and leads 
and sea ice patches in direct contact. Note that Figure 6 (bottom) shows a situation in August where the open water fraction due to ponds and leads is already large.

A data set on melt pond statistics [Fetterer et al., 2008] made available by the National Snow and Ice Data Center (NSIDC) was helpful for quantitative studies and for necessary assumptions about the shape of melt ponds and their typical size as a function of the sea ice concentration in the parametrizations derived in the next subsection. The data result from visible band imagery from high-resolution satellites over three Arctic Ocean sites in summer 1999 and over four sites in the summers of 2000 and 2001. From 101 images, melt pond statistics are available for 400 squares of $500 \mathrm{~m}$ by $500 \mathrm{~m}$ size. We used for our purposes only squares in which the surface consists of ice and ponds with a lead fraction smaller than $2 \%$ since characteristic sizes of leads were not available. These conditions were met in 234 squares between June and September during the three years.

At a first glance, open water structures in melt-pond-covered regions show a very irregular distribution with different shapes. Since only the number of ponds and the pond area are given in the observations but a certain assumption is necessary about the pond shape for deriving form drag (next subsection), we first used the available data to examine specific shape assumptions. The effect of two different shapes on the number density of floes is demonstrated in Figure 6, which shows a comparison of observed pond numbers with results of equation (38), below. There, we first assumed a quadratic and then a circular shape and used a linear dependence of the pond length (cross-wind dimension) $D_{w}^{\prime}$ on $A$, as described below.

The results hint that for $0.4<A<0.7$ a circular shape and for $A>0.7$ a quadratic shape is confirmed by the data. A possible reason is that ponds get often connected when 
they are growing so that deviations from a circular shape become larger with decreasing $A$. However, at the present stage of knowledge, we do not include this effect in the parametrization since the resulting curves are both within the scatter of the observations; we use here the most simple quadratic shape. Moreover, as clearly seen in Figure 1, real shapes of melt ponds and leads can differ from a quadratic or circular shape; but, as in the previous sections for the MIZ, it can be easily shown that other assumptions about the mean shapes would modify form drag again only by a constant factor.

\subsubsection{Derivation of drag coefficients}

The change in morphology over a melt-pond-covered surface causes a necessary change in the derivation of the form drag. It starts with equation (11) for the floe number density, which we replace for a pond or lead covered surface by

$$
\frac{N_{p}}{S_{t}}=\frac{A_{p}}{S_{p}}=\frac{1-A}{S_{p}}
$$

$S_{p}$ is the mean area of an individual pond or lead with

$$
S_{p}=c_{s}^{\prime} D_{w}^{\prime 2}
$$

$N_{p}$ is the number of ponds and leads in the domain of size $S_{t} . A_{p}$ is now the surface fraction of melt ponds and leads given by

$$
A_{p}=c_{s}^{\prime} \frac{N_{p} D_{w}^{\prime 2}}{S_{t}}
$$

where $D_{w}^{\prime}$ is the cross-wind dimension of the ponds and leads. As explained in the previous subsections, a difference in the shape factor $c_{s}^{\prime}$ for ponds to $c_{s}$ for floes is not crucial since $c_{s}^{\prime}$ occurs only as a constant factor in the parametrization formula of form drag. 
Analogous to equations (13) and (17), we obtain

$P_{d}=\frac{1}{N_{p} h_{p} D_{w}^{\prime}} N_{p} S_{c}^{\prime 2}\left[c_{w}^{\prime} \int_{0}^{D_{w}^{\prime}} \int_{z_{0, w}}^{h_{p}} \frac{\rho U^{2}}{2} d z d y\right]$

and

$\tau_{d}=\frac{1}{c_{s}^{\prime}} P_{d} \frac{h_{p}}{D_{w}^{\prime}}(1-A)$

where $h_{p}$ is now the elevation of the ice surface relative to the pond surface or to the open water surface in leads.

Finally, with all the above assumptions, we arrive at

$$
C_{d n 10}=(1-A) C_{d, w}+A C_{d, i}+\frac{c_{e}^{\prime}}{2}\left[\frac{\ln \left(h_{p} / z_{0, w}\right)}{\ln \left(10 / z_{0, w}\right)}\right]^{2} S_{c}^{\prime 2} \frac{h_{p}}{D_{w}^{\prime}}(1-A)
$$

with the effective coefficient of resistance

$$
c_{e}^{\prime}=\frac{c_{w}^{\prime}}{c_{s}^{\prime}}
$$

This equation differs from the corresponding equation for the MIZ in its dependence on $A$; and, in general, also $c_{e}^{\prime}$ and $S_{c}^{\prime}$ could be different. We stress that equation (43) leads to the same type of parametrization as in the MIZ (e.g., equation (35)) only when the aspect ratio $h_{p} / D_{w}^{\prime}$ is proportional to $A$. We investigate in the following under which assumptions such proportionality is valid.

\subsubsection{Parameterization of $h_{p}$ and melt pond length}

A least square fit of the Fetterer et al. [2008] data of melt pond size and width as a function of $A$ is given by

$$
D_{w}^{\prime}=D_{\min } A+D_{\max }(1-A)
$$


where $D_{\min }=2.26 \mathrm{~m}$ and $D_{\max }=24.63 \mathrm{~m}$ (Figure 6 ). This equation means that the initial pond size $D_{\min }$ is larger than zero. We can only speculate at present if this behavior for $A \rightarrow 1$ agrees with nature since the smallest resolvable pond size by the satellite was $1 \mathrm{~m}$. However, it is not unrealistic that an initial pond size exists since a floe surface is mostly uneven so that melt water will quickly flow together in the shallow small scale floe depressions.

Even so, we investigate for simplicity also the following assumption as an approximation of the above fit

$$
D_{w}^{\prime}=D_{\max }(1-A)
$$

with $D_{\max }=33 \mathrm{~m}$ (Figure 6). Both equations (45) and (46) would imply a maximum average pond size of roughly $30 \mathrm{~m}$. We do not consider this as critical since for small $A$ the surface morphology is better represented by the MIZ assumptions.

At present, we can only speculate about the best parametrization for $h_{p}$. One reason is that we do not have enough data for the derivation of a parametrization distinguishing between ponds and leads. In the case that the water fraction consists only of melt ponds, we assume that $h_{p}$ is proportional to $(1-A)^{\nu}$ for $A \rightarrow 1$. The dependence on $(1-A)^{\nu}$ is reasonable since a decrease in $A$ results first in an increase of melt pond depth [Skyllingstad et al., 2009], which in turn increases the distance between the surface of the melt water and the sea ice due to the differences in density. The power $\nu>0$ quantifies the rate of $h_{p}$ increase when $A$ decreases. But during further melting, sea ice thickness and hence $h_{p}$ decrease, which suggests a proportionality of $h_{p}$ to $A^{\mu}$ for $A \rightarrow 0$ with $\mu>0$. So, taking into account both limiting cases, a possible parametrization is

$$
h_{p}=h_{e} A^{\mu}(1-A)^{\nu}
$$


where $h_{e}$ is a length scale related to the freeboard of melt ponds.

For $\mu=\nu=1$, we obtain $h_{e}=4 h_{p, \max }$, where $h_{p, \max }$ is the maximum freeboard. Assuming $h_{p, \max } \approx 0.3 \mathrm{~m}( \pm 30 \%)$, the value of $h_{e}$ is $1.2 \mathrm{~m}( \pm 30 \%)$.

\subsubsection{Parametrization of the sheltering function $S_{c}$ for melt ponds}

For simplicity, we use the sheltering function (31), although the differences in the morphology between summer sea ice and sea ice in the MIZ could have an effect. For example, the curvature of the upstream floe edges is convex while that of ponds is concave when a circular shape is assumed for both floes and ponds. The presently available data do not allow such specification, but this could be another point for future improvements.

\subsection{5. $\mathrm{C}_{\mathrm{dn} 10}$ for the inner Arctic using different approximations}

Using equations (45) and (47) in equation (43) as well as the most simple parametrization of the sheltering function (equation 31), we obtain

$$
C_{d n 10}=(1-A) C_{d, w}+A C_{d, i}+\frac{c_{e}^{\prime}}{2}\left[\frac{\ln \left(h_{e} A^{\mu}(1-A)^{\nu} / z_{0, w}\right)}{\ln \left(10 / z_{0, w}\right)}\right]^{2} \frac{h_{e} A^{\mu}(1-A)^{1+\nu+1 /(10 \beta)}}{D_{\min } A+D_{\max }(1-A)} .
$$

This form differs in its $A$ dependence from the corresponding parametrizations for the MIZ (e.g., equation (35)).

The same structure of equations with respect to their $A$ dependence for the MIZ and melt ponds regime follows only when equations (46) and (47) are used for $D_{i}$ and $h_{p}$ in equation (43) so that

$C_{d n 10}=(1-A) C_{d, w}+A C_{d, i}+\frac{c_{w}}{2}\left[\frac{\ln \left(h_{e} A^{\mu}(1-A)^{\nu} / z_{0, w}\right)}{\ln \left(10 / z_{0, w}\right)}\right]^{2} \frac{h_{e}}{D_{\max }} A^{\mu}(1-A)^{1+\nu+1 /(10 \beta)}$. 
However, the AN10 equivalent parametrization (equation 6) follows when we assume a constant freeboard $\left(h_{p}=0.24 \mathrm{~m}\right)$ in the logarithm term.

With $c_{e}^{\prime}=0.3$ and $z_{0, w}=3.27 \cdot 10^{-4}$ in (49), one obtains

$$
C_{d n 10}=(1-A) C_{d, w}+A C_{d, i}+0.06 \frac{h_{e}}{D_{\max }} A^{\mu}(1-A)^{\nu+1 /(10 \beta)},
$$

since $0.06 h_{e} / D_{\max }=2.23 \cdot 10^{-3}$. In the most simple case $(\beta=1, \mu=\nu=1)$, the exponent $\nu+1 /(10 \beta)=1.1$ so that it produces only slightly different results than an exponent 1 .

Results of the parametrized drag coefficients obtained from equations (48), (49), and (50) with different parameter values $\mu$ and $\nu$ are shown together with the SHEBA observations in Figure 6. The observations and their accuracy are discussed in detail by AN10. They represent drag coefficients over a mixture of ice, leads, and melt ponds, while the modeled curves are strictly valid only for melt-pond-covered sea ice. Nevertheless, the parameterization results are in the range of the Atmospheric Surface Flux Group (ASFG) data. When we exclude the lowermost point for $A \approx 0.7$, the latter show on average a similar increase of drag coefficients with decreasing $A$ as the parametrization results. AN10 mention that the ASFG tower data, which show less scatter than the other data, are more reliable than the others since ASFG represents observations with several instruments mounted on a $20 \mathrm{~m}$ tower, while the others are obtained from 3 m masts with only one sonic anemometer.

Results of the modeled drag coefficients in the top graph of Figure 6 were all obtained with the parameter values $\beta=\mu=\nu=1$. With these values, results do not differ much from each other.

In the bottom graph, the parameter $h_{e}$ is chosen with the condition that the maximum $h_{p}$ is in all curves between 0.3 and $0.4 \mathrm{~m}$. Upper and lower reasonable limits of $\mu$ and 
$\nu$ are selected so that possible other curves with different values are in between the two pairs of curves shown. Obviously, the exponents $\mu$ and $\nu$ have a large impact on the structure of the $C_{d}$ curves. Results are not shown in the whole range of $A$ since we can expect that for small $A$ the surface morphology would change to the floe structure as in the MIZ; and, at present, summer data for drag coefficients are available only for melt pond and lead concentrations with $A>0.5$. Nevertheless, the data do not seem to be in contradiction to any of the curves obtained from the parametrizations. Additional data are required to get more insight into the real functional dependence of the drag coefficients on $A$ during summertime conditions. As long as such data are not available, the final parametrizations which we summarize in Section 5 will be based on the curves with convex curvature because it is less difficult to extrapolate them to the range $A<0.4$ by merging them with the parametrizations for the MIZ.

\section{Discussion of Parametrizations and their Region of Applicability}

Several parametrizations of the neutral drag coefficients over fractional sea ice cover have been derived in the previous sections; these differ in complexity. It has been shown that the AN10 formulation can be obtained from these parametrizations but only after introducing simplifying assumptions in the basic formulations.

Before we discuss more general questions concerning the applicability and relevance of the new parametrizations, we summarize in the following what has been achieved by the new parametrizations relative to the previous ones of HBG88, LB05, and Birnbaum and Lüpkes [2002], who followed a similar line as in the present work.

One of the most important differences between previous approaches and the present one is that only ours gives the possibility to distinguish different ice morphologies. Although 
the parametrization by HBG88, LB05, as well as Birnbaum and Lüpkes [2002] include a dependence on floe lengths, freeboard, and distances between floes, they are related only to MIZ conditions.

Another difference is that the derivation of the new formulae needs fewer restrictive assumptions concerning the distribution of floes. We consider this an important point since it shows that the range of validity of the resulting equations is broader than for the restrictive previous models. We explain this in the following in more detail.

Different from our present model, HBG88 used a 2D concept based on a periodic floe distribution (not explicitly mentioned in their work) in which they replace right from the beginning of their derivation $A$ by $D_{i} /\left(D_{w}+D_{i}\right)$. The corresponding equation for 3D floes is equation (29), which is used in the derivation of the present model only to specify one of the possible sheltering functions. Thus, different from the present model, in HBG88 $A$ is not present in their final formulation of surface drag. Furthermore, the values of $D_{i}$ and $D_{w}$ are not parametrized as a function of $A$, which had been proposed by Mai [1995] and which was done first by Birnbaum and Lüpkes [2002]. Only the introduction of sheltering required an assumption about the floe distribution, which is the main point where the assumption of $3 \mathrm{D}$ instead of $2 \mathrm{D}$ floes has an impact on the equations. But we have shown that the impact of sheltering on the drag coefficients is mostly small and that by introducing the sheltering functions (30) and (31) instead of (28), as used by HBG88 and LB05, the distance between floes no longer occurs as a parameter. This means that in the present parametrization the assumption of a periodic floe distribution could finally be avoided. 
At this point, it is also important to mention that the irregular shape of floes, melt ponds, and leads - not included in the previous work - can be accounted for in the new parametrization by a shape parameter $c_{s}$.

Another important difference between our present parametrization and the LB05 model is the use of different functions for the floe parameters as functions of $A$. The new functions are based on a larger data set and we introduced the parameter $\beta$ in the $D_{i}$ function; variability in both can explain the scatter in the observed form drag. With the modified functions, a singularity in the dependence of $D_{i}$ on $A$ could be avoided.

The new scheme allows us to propose a hierarchy of parametrization levels for different kinds of models. Before explaining this hierarchy in section 5, we discuss in the following more general questions concerning the derived drag parametrizations. These questions are:

Is the present data base large enough to represent the average conditions over regions with fractional sea ice and can the parameters occuring in the parametrization equations be constrained? Is it important to account for the variability of surface drag caused by sea ice variability? Does the same parametrization hold in a changing climate with changing sea ice?

With regard to the first question, it is important to understand that the observations shown in the previous figures are not all of the same significance since some of them represent only one experiment or were obtained at only one location. Most comprehensive is the SHEBA data base since data were sampled over the whole summer at several masts. These data point to a large variability of roughly $\pm 50 \%$ for the drag coefficients in a small region of about $15 \mathrm{~km}$ diameter. The reliability of this scatter is an open question (see 
previous section), but similar variability was found by aircraft observations over a region with complete sea ice cover [Garbrecht et al., 2002].

Over the MIZ, data from various campaigns suggest an even larger variability of drag coefficients than in the inner summertime Arctic. A comparison of REFLEX data with other data shows large differences in the MIZ, especially at large ice concentrations. LB05 supposed that differences between REFLEX and MIZEX data were caused by different ice regimes in the eastern Fram Strait MIZ and western Fram Strait, where more shear and rougher ice are expected. But a final conclusion would require additional measurements.

Similar variability exists for the inner Arctic regions. Practically, the presently available data base is not large enough to clearly identify geographic regions with different drag coefficients, but the existence of regimes with larger or smaller drag coefficients than those observed during SHEBA cannot be excluded. For example, the distance to coasts might play an important role, and roughness regimes could change similarly as the ice thickness regimes differ in the western and eastern Arctic [Steiner, 2001]. Combining all data in one figure hints to, on average, slightly lower drag coefficients in the inner summertime Arctic than in the MIZ (see Figure 6) and to a stronger variability in the MIZ.

After all, the presently available information about the governing parameters (diameters of floes and melt ponds, freeboard, and skin drag for $A=1$ and $A=0$ ) determining the $A$ dependence of the neutral drag coefficient does not allow further constraining the allowed range of these parameters. The recommended values and uncertainties, as already addressed in the previous sections and compiled in Section 5, reflect the presently available knowledge. A further refinement would only be possible with new observations which should combine the necessary meteorological and sea ice information. However, a test of 
the derived parametrizations in climate models and an assessment of the model's sensitivity to different parameter combinations could allow further conclusions on the necessary accuracy of the parameters.

The second question concerning the importance of the drag variability addresses also the scale of the atmospheric or sea ice models used. Both data sets used by AN10 and based on REFLEX represent average data which are relevant for large scale models. With smaller grid sizes, deviations from the mean and hence the scatter in the observations become important. The parametrizations derived here are able to account for this scatter provided that the parameters floe size, pond and lead size, freeboard, and skin drag of ice and water are given. So, the second question could be answered in the future by varying these parameters in a sensitivity study with a coupled ice-ocean-atmosphere model. But already studies like that of Vihma et al. [2003] and Birnbaum and Lüpkes [2002] hint to a large impact of drag variability on atmospheric model results.

As Figure 6 shows, the addition of form drag can alter the maximum surface drag by a factor 1.3 if the parameters are chosen as in the lowermost curve and by a factor 2.2 for parameters like in the uppermost curve. Previous parametrizations accounting for form drag in a simple way, so that they could be implemented in climate models, focused on the MIZ only. When this type of parametrizations is available now for the whole Arctic, the additional drag might influence both sea ice drift [Zhang and Rothrock, 2003] and ABL characteristics. The latter has been shown in a modeling study over a region near Svalbard by Vihma et et al. [2003], where the inclusion of the form drag by ridges affected the ABL height and thus the profiles of wind speed and temperature. 
Finally, it should be mentioned that the large variability of floe distributions in nature hint at the limit of the simplest proposed formulae in which the drag coefficients depends mainly on $A$ (or $A(1-A)$ in case of the MIZ). For example, during on-ice winds, situations can occur with densely packed small floes in the MIZ and thus a large number of floe edges per unit area. For such situations, the most complex formulae derived here would be of advantage provided that it is possible to predict $D_{i}$ and $h_{f}$ correctly.

As concerns the last question, the drag parametrizations derived here could be used in climate models with some of the parameters being adjusted to modeled quantities. For instance, even if no ice is included, the present formulae allow adjusting the skin drag over open water dependent on the friction velocity using, for example, a Charnock-type formula. A coupled sea-ice-atmosphere-climate or sea-ice-ocean model could make use of the $C_{d}$ equations containing explicitly the sea ice freeboard. This would allow investigating the modified drag in a scenario with strongly decreasing sea ice thickness. The most sophisticated numerical atmosphere-ice-ocean models might even be able to predict floe, pond, and lead sizes. Attempts in this direction have been made by Birnbaum [1998]. Also Steiner [2001] studied the form drag derived from a sea ice model. Moreover, different skin drags could be used depending on the region with larger values in regions where much shear can be expected. LB05 showed that, in the western part of the Fram Strait MIZ, values up to $C_{d, i}=2.9 \cdot 10^{-3}$ can be justified to reproduce MIZEX observations, while the present finding suggets lower values $\left(C_{d, i}=1.5 \cdot 10^{-3}\right)$ for the northwestern part of Fram Strait and the inner summer Arctic. 


\section{Recommendations for the Application of the Parametrizations}

The question arises how to use the new parametrizations in weather prediction and climate models. Based on the findings of the previous sections, we suggest using different parametrizations of $C_{d n 10}$ depending on the physical complexity of the atmosphere-iceocean model used and its horizontal resolution. For simplicity, we call this model the background model in the following compilation of suggested parametrizations. The basic concept is always the drag partitioning, namely equation (2). The proposed parametrizations are different for the marginal sea ice zone and the summertime inner Arctic.

\subsection{Equation Summary for the Marginal Sea Ice Zone}

We distinguish between three levels of complexity of the background model.

\section{Level 1:}

The most complex background model solves prognostic equations for ice thickness (or volume) and $D_{i}$. In this case, we suggest using

$$
\begin{aligned}
C_{d, f} & =\frac{c_{e}}{2}\left[\frac{\ln \left(h_{f} / z_{0, w}\right)}{\ln \left(10 / z_{0, w}\right)}\right]^{2} S_{c}^{2} \frac{h_{f}}{D_{i}} A \\
S_{c}^{2} & =1-\exp \left[-s_{l} \beta(1-A)\right] .
\end{aligned}
$$

In the REFLEX observations, $h_{f}$ was about twice the value of the freeboard of level ice due to the occurrence of ridges on the floes. Since ridges are usually not predicted, this assumption is a rough but practicable method to obtain $h_{f}$ from a model predicting ice thickness. We recommend $c_{e}=0.3$, but a variation by $\pm 20 \%$ does not result in large differences for $C_{d n 10}$ since form drag is mostly not larger than $50 \%$ of the total drag. In the $S_{c}$ equation, we propose using $\beta=1$ and $s_{l}=22$. A variation of the product $\beta s_{l}$ by 
$\pm 30 \%$ changes the maximum drag by 1-5\% depending on the characteristic floe size. $z_{0, w}$ can be determined by the Charnock [1955] relation $\left(z_{0, w}=\alpha u_{\star}^{2} / g\right.$, where $\alpha=0.018[W u$, 1980] is constant).

Summing up, we have the tuning constants $z_{0, w}, c_{e}, s_{l}$, and $\beta$.

\section{Level 2}

In the case that freeboard $h_{f}$ is available from the background model, but not the floe length $D_{i}$, the parametrization

$$
\begin{aligned}
C_{d, f} & =\frac{c_{e}}{2}\left[\frac{\ln \left(h_{f} / z_{0, w}\right)}{\ln \left(10 / z_{0, w}\right)}\right]^{2} S_{c}^{2} \frac{h_{f}}{D_{\min }}\left(\frac{A_{\star}-A}{A_{\star}}\right)^{\beta} \\
A_{\star} & =\frac{1}{1-\left(D_{\min } / D_{\max }\right)^{1 / \beta}}
\end{aligned}
$$

can be used with $S_{c}$ given by equation (52). If $h_{f}$ is also not predicted, it can be approximated by equation (24) with the optimum values $h_{\min }=0.2 \mathrm{~m}, h_{\max }=0.55 \mathrm{~m}$, $D_{\min }=8 \mathrm{~m}$, and $D_{\max }=300 \mathrm{~m}$. A variability by $\pm 20 \%$ of the latter values changes the maximum drag coefficients roughly by the same percentage. For REFLEX data, which were representative for the northeastern part of the Fram Strait, $\beta=1.4$ was most suitable; in the western Fram Strait, the observations were best represented using $\beta=0.3$, when the same prescribed value of skin drag is used (see below). As long as only few data are available from other MIZ regions than the Fram Strait, a possible compromise is to use $\beta=1$. A $\beta$ variation by \pm 0.2 causes a variability of the maximum $C_{d n 10}$ by less than $10 \%$.

On this level, we have the tuning constants $z_{0, w}, c_{e}, s_{l}, \beta, h_{\min }, h_{\max }$. We recommend not modifying $D_{\min }$ and $D_{\max }$ by more than $\pm 20 \%$ from the values given. 


\section{Level 3}

The level 2 scheme can be once more simplified for those model applications which need to save as much cpu time as possible and require a very efficient drag parametrization. Here the AN10-type parametrization is of advantage, which means that $A_{\star}$ is replaced by one, and $h_{f}$ is approximated by a constant. The first assumption allows also replacing the sheltering function by 1 , as has been explained. With $h_{f}=0.41 \mathrm{~m}$ and $D_{\min }=8 \mathrm{~m}$, our suggestion reads

$$
C_{d, f}=7.68 \cdot 10^{-3}\left[\frac{\ln \left(0.41 / z_{0, w}\right)}{\ln \left(10 / z_{0, w}\right)}\right]^{2}(1-A)^{\beta} A
$$

$\beta$ has the same values as in the level 2 approximation.

On this level, we have the tuning constants $z_{0, w}$ and $\beta$.

\section{Level 4}

Inserting $z_{0, w}=3.27 \cdot 10^{-4}$ in equation (55), we obtain

$$
C_{d, f}=3.67 \cdot 10^{-3}(1-A)^{\beta} A
$$

which is the AN10 (equation (7)) analoguous parametrization for the MIZ.

There is one tuning constant $\beta$ for this level.

The limitations of the approaches (55) and (56) were discussed in section 3.1.5.

\section{Skin drag}

In all equations (51), (53), and (55), Charnock's formula can be used for calculating $z_{0, w}$. The latter can be used also to calculate the skin drag coefficient of open water as $C_{d, w}=\kappa^{2} /\left[\ln \left(z_{p} / z_{0, w}\right)\right]^{2}\left(z_{p}=10 \mathrm{~m}, \kappa=\right.$ von Karman constant $)$. This guarantees 
a smooth transition from the drag coefficients over the MIZ to the open ocean values. Allowing this variability has furthermore the advantage compared with the prescription of constant values that it could probably partly explain the variability of drag coefficients observed over the MIZ at low ice concentrations in different campaigns. Results in Figure 6 were obtained with $C_{d, w}=1.5 \cdot 10^{-3}$.

During winter, we suggest using $C_{d i}=1.6 \cdot 10^{-3}$ while keeping in mind that also much higher values occur sometimes in nature (LB05).

\subsection{Equation Summary for the Inner Summer Arctic}

The previous sections have shown that the morphology of summer sea ice influences strongly the functional dependence $C_{d}(A)$. For the present study, observations of drag coefficients and melt ponds were available only for $A>0.5$. This limit specifies the range of applicability of the following parametrizations, which were derived for melt-pond-covered sea ice; but the comparison with data showed that they represent conditions with ponds and leads as well.

\section{Level 1 and 2}

The parametrization suitable for the most complex background model, which delivers both freeboard $h_{p}$ and characteristic length of open water $D_{w}^{\prime}$, is

$$
C_{d, f}=\frac{c_{e}^{\prime}}{2}\left[\frac{\ln \left(h_{p} / z_{0, w}\right)}{\ln \left(10 / z_{0, w}\right)}\right]^{2} \frac{h_{p}}{D_{w}^{\prime}} S_{c}^{\prime 2}(1-A) .
$$

with $c_{e}^{\prime}=0.3$ and $S_{c}^{\prime 2}$ is given by equation (31) and depends only on $A$. The accuracy of this parametrization depends on the knowledge of $D_{w}^{\prime}$ and $h_{p}$. We do not specify a level 2 version as for the MIZ since, at present, we are not aware of a model predicting $D_{w}^{\prime}$. 
This parametrization has three tuning parameters: $z_{0, w}, c_{e}^{\prime}$, and $\beta$ in the sheltering function.

\section{Level 3}

In the case that both $h_{p}$ and $D_{w}^{\prime}$ are not obtained from a background model, we propose the parametrization based on data from melt-pond-covered surfaces, which resulted with $\beta=1$ used in $S_{c}^{\prime}$ as

$$
C_{d, f}=\frac{c_{e}^{\prime}}{2}\left[\frac{\ln \left(h_{e} A^{\mu}(1-A)^{\nu} / z_{0, w}\right)}{\ln \left(10 / z_{0, w}\right)}\right]^{2} \frac{h_{e} A^{\mu}(1-A)^{\nu+1.1}}{D_{\min } A+D_{\max }(1-A)},
$$

with $c_{e}^{\prime}=0.3, h_{e}=1.2 \mathrm{~m}, D_{\min }=2.26 \mathrm{~m}, D_{\max }=24.63 \mathrm{~m}$, and $\mu=\nu=1$.

This parametrization has the tuning parameters $c_{e}^{\prime}, z_{0, w}$, as well as $D_{\max }, D_{\min }$, and $h_{e}$.

\section{Level 4}

We found that under several simplifying assumptions this equation can be replaced by

$$
C_{d, f}=2.23 \cdot 10^{-3} A(1-A)^{1.1}
$$

On this level, there are no tuning parameters.

\section{Skin drag}

Skin drag for open water can still be variable in all formulae related to the inner Arctic. For example, $z_{0, w}$ can be again obtained from the Charnock formula or from any other concept. Skin drag over ice $\left(C_{d, i}\right)$ can vary depending on any small scale surface characteristic which is not accounted for by the form drag parametrization. We propose to use the skin drag coefficient $C_{d, i}=1.4 \cdot 10^{-3}$ for ice, as in AN10, and $C_{d, w}=1.5 \cdot 10^{-3}$ when 
the Charnock formula cannot be applied (e.g., in simple models which have no knowledge of the friction velocity).

\subsection{Application to Models, Tuning Constants}

When the above parametrizations are applied to climate models, different questions may arise as, for example, how to connect the different formulae for the MIZ and inner polar regions.

A rough distinction can first be made by considering the different ice conditions in the Arctic and Antarctic. In the Antarctic, melt ponds are rare. Thus, the MIZ formulae could be applied in all seasons and also in the inner regions to account for form drag of floe edges in cases with $A<1$. For large $A$, a combination is possible with parametrizations like that of Overland [1985], who applied different skin drag coefficients depending on the ice type.

In the winter Arctic, we also suggest using the formulae for the MIZ in the whole sea ice covered region. By this method, the drag coefficients might be underestimated in the inner Arctic regions since they do not explicitly account for the additional form drag by ridges. However, compared with the presently used drag coefficients in climate models, which do not account for form drag at all, there is no disadvantage of this method.

For the summer Arctic, we suggest using the formulae for melt pond and lead covered regions only in the inner regions; while in the MIZ (band of approximately 60-80 km width along the pack ice edges), the formulae based on REFLEX observations could be used. These observations were carried out during early spring and early autumn with varying ice conditions so that on the basis of present knowledge we assume that they are valid during 
the whole year. As for a backgrond model in which $h_{f}$ is predicted, this uncertainty does not occur because predicting $h_{f}$ accounts for any seasonal variability.

In our own experience with applying drag parametrizations to mesoscale models [Birnbaum and Lüpkes, 2002; Vihma et al., 2003], the use of different drag coefficients in neigboring grid cells is not problematic. However, it might be difficult in climate models to switch from the winter time formulae to the summer time parametrizations. We can only suggest here that this change should occur after the $10-\mathrm{m}$ temperature is above $-2^{\circ} \mathrm{C}$. Problems can perhaps be expected when $h_{f}$ is not predicted by the background model. In this case, at the initial stage of melting, an interpolation of drag coefficients obtained by the MIZ and inner Arctic formulae could be helpful to avoid numerical difficulties.

We selected some tuning constants in the above formulae but suggested also values for these constants which lead to the best agreement with the available observations. A tuning could, however, be useful to study a model's sensitivity to different ice conditions. As most important for the MIZ, we consider the parameter $\beta$, whose variation is best suited to study the impact of different floe size distributions on form drag. A smaller sensitivity was found for $h_{f}$; while the parameter $c_{e}$, which accounts for the shape effect, has again larger impact. Another important parameter is the skin drag coefficient. We have suggested here the value based on the observations over closed sea ice of the northeastern Fram Strait. However, as pointed out by LB05, larger values might be suitable for the western Fram Strait. Larger skin drag over ice causes a shift in $C_{d n 10}$ to larger values, which would better agree with MIZEX observations for high values of ice concentration.

For the inner Arctic, the shape parameter $c_{e}^{\prime}$ could be modified. Probably, the assumption of quadratic ponds and leads is a larger simplification than the same assumption for 
floes so that a modification of this parameter can be justified. Also other tuning parameters given above could be modified, but we suggest restricting the variation to $\pm 20 \%$ of the given values. We did not mention $\mu$ and $\nu$ as tuning parameters but recommend keeping both unchanged to avoid a convex curvature of the curve related to the parametrized drag coefficient, which might result in practical problems for low ice concentrations.

\section{Conclusions}

The main goal of the present work was to study the neutral atmospheric drag coefficient over regions with different sea ice morphology. Parametrizations were derived based on physical concepts rather than by fitting results of a complex model, as in Lüpkes and Birnbaum [2005], or by fitting observations, as in Andreas et al. [2010]. A hierarchy of formulae with different complexity resulted which can be applied to climate and weather prediction models as well as to sea ice models.

The basic idea for the derivation consisted in the partitioning of the total drag into contributions of skin drag and form drag caused by the edges of floes. As a first step, it was shown that the Andreas et al. [2010] polynomial fit can also be formulated in terms of the drag partitioning concept. The advantage of the parametrizations derived here is that they can be adapted to different roughness regimes provided that the morphological sea ice parameters concentration, ice freeboard, and characteristic floe and pond diameters are given. The dependence of a parametrization on such parameters is especially interesting with respect to future climate scenarios in which sea ice morphology and thus sea ice roughness and, as a consequence, sea ice drift could change.

It was shown that the variability of drag coefficients observed over the MIZ and summer inner Arctic can be explained by variability in the form drag. This was different in the 
model derived by Lüpkes and Birnbaum [2005], who explained extreme values of drag coefficients in the Fram Strait MIZ only as an effect of skin drag variability. In some of the previous studies, a decrease of drag coefficients at sea ice concentrations larger than about $50 \%$ was explained as the effect of flow sheltering downstream of ice floes. We showed, however, that in the parameter range of most observations the impact of sheltering is of minor importance and that the decreasing values of drag coefficients with increasing sea ice concentration are mainly an effect of floe geometry and its dependence on sea ice concentration.

Andreas et al. [2010] approximated drag coefficients in the MIZ and summertime Arctic by the same curve with the same functional dependence on the sea ice concentration and explained the increase of drag coefficients during sea ice melt as the effect of form drag due to elevated sea ice relative to the melt pond and lead surfaces. Our analytical model shows that the parametrization by a single curve applied to both ice regimes is possible for average conditions; but, in general, differences in the sea ice morphology between the MIZ and summertime Arctic can lead to different functional dependencies of drag coefficients on the sea ice concentration. We expect that locally the variability of drag coefficients might have a large influence on sea ice drift and atmospheric fluxes so that the more complex formulae derived here are especially important for high-resolution regional climate models. An increased number of observations by aircraft and during drifting stations could be helpful in the future to identify the regional regimes of surface drag and to specify the regional dependence of key parameters in the derived parametrizations.

Including the new parametrizations in climate models can be considered as a step forward relative to the prescription of only one constant roughness length for sea ice and 
for open water. Furthermore, testing the sensitivity of atmospheric variables on the parametrizations in climate models can lead to new findings about air-ice interaction processes.

The proposed formulae cannot solve all open questions related to the parametrization of surface roughness of sea ice covered regions. Most important for the future development is probably the combination of the present parametrizations with parametrizations accounting for the effect of pressure ridges in regions with closed sea ice cover.

The present paper aimed only at parametrizing the neutral effective drag coefficient over a mixture of ice and water. The inclusion of stability, which strongly influences surface drag, for example, during winter in the close environment of leads [Lüpkes et al., 2008], requires future work. One possible strategy is described by Lüpkes and Birnbaum [2005]. Their basic idea (their chapter 4) was to use the effective roughness length based on the parametrized neutral drag coefficients together with Monin-Obukhov theory for stability corrections. Another strategy was proposed by Andreas et al. [2010]. The quantification of the stability effect dependent on ice morphology will be addressed in future work. 


\section{Appendix A: List of symbols}

$A$ - sea ice fraction

$A_{\star}-$ dimensionless constant in equation (26)

$A_{p}-$ concentration of pond and lead covered surface

$\alpha_{r}-$ floe aspect ratio $D_{i} / h_{f}$

$\beta$ - constant exponent describing the dependence of $D_{i}$ on $A$

$c_{s}, c_{s}^{\prime}$ - shape parameters for floes and melt ponds $\left({ }^{\prime}\right)$

$c_{w}-$ coefficient of resistance of an individual floe

$c_{e}, c_{e}^{\prime}-c_{w} / c_{s}-$ effective resistance coefficients

$C_{d, f}-$ form drag coefficient

$C_{d, f \max }-$ maximum value of $C_{d, f}$

$C_{f}$ - part of form drag which is independent on $A$

$C_{d n 10}-$ neutral drag coefficient at $10 \mathrm{~m}$ height

$C_{d, i}-$ neutral skin drag coefficient at $10 \mathrm{~m}$ height over ice

$C_{d, w}-$ neutral skin drag coefficient at $10 \mathrm{~m}$ height over water

$D_{i}-$ cross wind dimension of a floe, $(L)$

$D_{\min }, D_{\max }-$ minimum and maximum ice floe length $(L)$

$D_{w}-$ distance between floes, $(L)$

$D_{w}^{\prime}-$ cross wind dimension of a melt pond or lead, $(L)$

$h_{\max }, h_{\min }-$ maximum and minimum ice freeboard $(L)$

$h_{f}-$ freeboard of ice floes $(L)$ 
$h_{f, l}$ - linear fit of $h_{f}(L)$

$h_{f, c}-$ characteristic constant value approximating $h_{f, l}(L)$

$h_{p}$ - elevation of ice surface relative to the water surface in ponds or leads $(L)$

$h_{e}$ - length scale related to the freeboard of melt ponds $(L)$

$N-$ number of floes

$n$ - number density of floes $\left(\mathrm{L}^{-2}\right)$

$N_{p}-$ number of ponds and leads

$P-$ dynamic pressure $\left(M T^{-2} L^{-1}\right)$

$\rho-$ air density $\left(M L^{-3}\right)$

$S_{c}, S_{c}^{\prime}-$ sheltering function

$s_{l}-$ constant in the sheltering function

$S_{t}$ - domain area with $N$ floes or with ponds and leads $L^{2}$

$S_{i}$ - area of a single floe $\left(L^{2}\right)$

$S_{p}-$ area of an individual pond or lead $\left(L^{2}\right)$

$\tau_{d}-$ momentum flux $\left(M L^{-1} T^{-2}\right)$

$U$ - horizontal height dependent wind speed $\left(L T^{-1}\right)$

$U_{10}-$ horizontal $10-\mathrm{m}$ wind speed $\left(L T^{-1}\right)$

$z_{0, w}, z_{0, i}$ - roughness length over water and ice $(L)$

\section{Appendix B: A model with quasi-empirical form drag}

It is shown in the following how the AN10 parameterization (equation (1)) can be written in terms of equation (2). This goal is achieved with the assumption that $C_{d n 10}$ in equation 
(2) can be fitted by a quadratic $C_{d n 10}=\alpha+\beta A+\gamma A^{2}$, where $\alpha, \beta$, and $\gamma$ are tuning constants. So, $C_{d, f}$ can be written as the difference between total drag and skin drag as

$$
C_{d, f}(A)=\alpha+\beta A+\gamma A^{2}-(1-A) C_{d, w}-A C_{d, i}
$$

For a determination of the constants, we apply three constraints, namely $C_{d, f}(0)=0$, $C_{d, f}(1)=0$, and $C_{d, f}\left(A_{m}\right)=C_{d, f \max }$, where $C_{d, f \max }$ is the maximum form drag coefficient. After straightforward algebraic operations, one finds that $A_{m}=0.5$ and

$$
\left.\begin{array}{rl}
\alpha & =C_{d, w} \\
\beta & =C_{d, i}-C_{d, w}+4 C_{d, f \max } \\
\gamma & =-4 C_{d, f \max }
\end{array}\right\}
$$

After substituting constants (B2) into (B1), we obtain

$$
C_{d, f}=4 C_{d, f \max } A(1-A)
$$

and finally, after adding skin drag, equation (6), which obviously is a second order polynomial

$$
C_{d n 10}=C_{d, w}+\left(4 C_{d, f \max }+C_{d, i}-C_{d, w}\right) A-4 C_{d, f \max } A^{2}
$$

With $C_{d, w}=1.4 \cdot 10^{-3}, C_{d, i}=1.5 \cdot 10^{-3}, C_{d, f \max }=0.558 \cdot 10^{-3}$, equation (B4) is identical with the AN10 equation (1).

Acknowledgments. We thank NSIDC for the data on melt pond statistics [Fetterer et al., 2008]. Constructive comments of three anonymous reviewers helped to improve the text. We also thank G. Birnbaum, H. Borth, A. Grachev, S. Danilov, and D. Sidorenko for discussions of several questions related to this work. This work contributes to the project MiKlip funded by the German Federal Ministry of Education and Research (FKZ: 01LP1126A). A part of this work was also funded by DFG (Grant No. LU 818/3-1). 
The U.S. National Science Foundation supported Ed Andreas's participation in this work through award 10-19322.

\section{References}

Anderson, R.J. (1987), Wind stress measurements over rough sea ice during the 1984 Marginal Ice Zone Experiment', J. Geophys. Res., 92(C7), 6933-6941.

Andreas, E.L (1995), Air-ice drag coefficients in the western Weddell Sea. 2. A model based on form drag and drifting snow. J. Geophys. Res., 100 (C3), 4833-4843.

Andreas, E.L, W.B. Tucker III, and S.F. Ackley (1984), Atmospheric boundary-layer modification, drag coefficient, and surface heat flux in the Antarctic marginal ice zone, J. Geophys. Res., 89 (C1), 649-661.

Andreas, E.L, T.W. Horst, A.A. Grachev, P.O.G. Persson, C.W. Fairall, P.S. Guest, and R.E. Jordan (2010), Parametrizing turbulent exchange over summer sea ice and the marginal ice zone, Q.J.R. Meteorol. Soc., 138, 927-943.

Banke, E.G., and S.D. Smith (1975), Measurement of form drag on ice ridges, AIDJEX Bull., 28, 21-27.

Birnbaum, G. (1998), Numerical modelling of the interaction between atmosphere and sea ice in the Arctic maginal ice zone, Reports on Polar Research, 268, Alfred Wegener Institute, Bremerhaven, 160 pp.

Birnbaum, G., and C. Lüpkes (2002), A new parametrisation of surface drag in the marginal sea ice zone, Tellus A, 54A(1), 107-123.

Charnock, H. (1955), Wind stress over a water surface, Quart. J. Roy. Meteorol. Soc., 81, 639-640. 
Claussen, M. (1990), Area-averaging of surface fluxes in a neutrally stratified, horizontally inhomogeneous atmospheric boundary layer, Atmos. Environ., 24A, 1349-1360.

Fairall, C.W., and R. Markson (1987), Mesoscale variations in surface stress, heat fluxes, and drag coefficient in the marginal ice zone during the 1983 marginal ice zone experiment, J. Geophys. Res., 92, 6921-6932.

Fetterer, F., S. Wilds, and J. Sloan (2008), Arctic sea ice melt pond statistics and maps, 1999-2001, Boulder, Colorado USA: National Snow and Ice Data Center. Digital media.

Garbrecht, T., C. Lüpkes, J. Hartmann, and M. Wolff, (2002), Atmospheric drag coefficients over sea ice - validation of a parametrisation concept, Tellus A, 54(2), 205-219.

Garbrecht, T., C. Lüpkes, E. Augstein, C. Wamser (1999), Influence of a sea ice ridge on low-level airflow, J. Geophys. Res., 104 (D20), 24,499-24,507.

Grötzner, A., R. Sausen, and M. Claussen (1996), The impact of sub-grid scale seaice inhomogeneities on the performance of the atmospheric general circulation model ECHAM3, Clim. Dyn., 12, 477-496.

Guest, P.S., and K.L. Davidson (1987), The effect of observed ice conditions on the drag coefficient in the summer east greenland sea marginal ice zone, J. Geophys. Res., 92(C7), 6943-6954.

Hanssen-Bauer, I. and Y.T. Gjessing (1988), Observations and model calculations of aerodynamic drag on sea ice in the Fram Strait, Tellus, 40A, 151-161.

Hartmann, J., C. Kottmeier, and C. Wamser (1992), Radiation and eddy flux experiment 1991 (REFLEX I), Reports on Polar Research, 105, Alfred Wegener Institute, Bremerhaven, 72 pp. 
Hartmann, J., C. Kottmeier, C. Wamser, and E. Augstein (1994), Aircraft measured atmospheric momentum, heat and radiation fluxes over Arctic sea ice, in O.M. Johannessen, Muench,R.D., and J.E. Overland (ed.) The polar oceans and their role in shaping the global environment, American Geophysical Union, Washington, DC, 443-454.

Inoue, J., M. Wakatsuchi, and Y. Fujiyoshi (2004), Ice floe distribution in the Sea of Okhotsk in the period when sea-ice extent is advancing, Geophys. Res. Lett., 31, L20303, doi:10.1029/2004GL020809.

Kottmeier, C., J. Hartmann, C. Wamser, A. Bochert, C. Lüpkes, D. Freese, and W. Cohrs (1994), Radiation and eddy flux experiment 1993 (REFLEX II), Reports on Polar Research, 133, Alfred Wegener Institute, Bremerhaven, 62 pp.

Lopez, S., C. Lüpkes, and K.H. Schlünzen (2005), The effect of different k- $\epsilon$ closures on the results of a micro-scale model for the flow in the obstacle layer, Meteorol. Z., 14(6), 839-848, doi:10.1127/0941-2948/2005/0084.

Lu, P., Z. Li, B. Chen, and M. Leppäranta (2011), A parameterization of the ice-ocean drag coefficient, J. Geophys. Res., 116, C07019, doi:10.1029/2010JC006878.

Lüpkes, C., Vihma, T., Birnbaum, G., Dierer, S., Garbrecht, T., Gryanik, V., Gryschka, M., Hartmann, J., Heinemann, G., Kaleschke, L., Raasch, S., Savijärvi, H., Schlünzen, K., and Wacker, U. (2012), Mesoscale modelling of the Arctic atmospheric boundary layer and its interaction with sea ice, Chapter 7 in: ARCTIC climate change - The ACSYS decade and beyond (P. Lemke and H.-W. Jacobi (Edts.)) Springer, Atmospheric and Oceanographic Sciences Library, 43, doi:10.1007/978-94-007-2027-5.

Lüpkes, C., Gryanik, V., Witha, B., Gryschka, M., Raasch, S., and Gollnik, T. (2008), Modeling convection over arctic leads with LES and a non-eddy-resolving microscale 
model, J. Geophys. Res., 113, C09028, doi:10.1029/2007JC004099.

Lüpkes, C., and G. Birnbaum (2005), Surface drag in the Arctic marginal sea-ice zone: a comparison of different parameterisation concepts, Boundary Layer Meteorol., 117, 179-211.

Mai, S. (1995), Beziehungen zwischen geometrischer und aerodynamischer Oberflächenrauhigkeit arktischer Meereisflächen, M.S. Thesis, University of Bremen, 75 pp.

Mai, S., C. Wamser, and C. Kottmeier (1996), Geometric and aerodynamic roughness of sea ice, Boundary-Layer Meteorol., 77, 233-248.

Overland, J.E. (1985), Atmospheric boundary layer structure and drag coefficients over sea ice, J. Geophys. Res., 90, 9029-9049.

Raupach, M.R., (1992), Wind drag partition on rough surfaces, Boundary Layer Meteorol., $60,375-395$.

Roeckner, E., et al. (2003), The atmospheric general circulation model ECHAM5. Part 1. Model description, MPI Rep., 349, Max Planck Inst. for Meteorol., Hamburg, Germany.

Schlichting, H. (1936), Experimentelle Untersuchungen zum Rauhigkeitsproblem, Ingenieur-Archiv, 7, 1-34.

Schröder, D., T. Vihma, A. Kerber, and B. Brümmer (2003), On the parametrisation of turbulent surface fluxes over heterogeneous sea ice surfaces, J. Geophys. Res., 108 (C6), 3195, doi:10.1029/2002JC001385

Shao, Y., and Y. Yang (2008), A theory for drag partition over rough surfaces, J. Geophys. Res., 113, F02S05, doi:10.1029/2007JF000791. 
Skamarock WC, J.B., Klemp , J. Dudhia, D.O., Gill, D.M. Barker, M.G. Duda, X.-Y. Huang, W. Wang, and J.G. Powers (2008), A description of the advanced research WRF version 3. NCAR Technical Note - 475+STR, Boulder Colorado, USA, 113 pp.

Skyllingsstad, E.D., C.A. Paulson, and D.K. Perovich (2009), Simulation of melt pond evolution on level ice, J. Geophys. Res., 114, C12019, doi:10.1029/JC005363

Steiner, N. (2001), Introduction of variable drag coefficients into sea-ice models, Annals of Glaciology, 33, 181-186.

Stössel, A. and M. Claussen (1993), On the momentum forcing of a large-scale sea-ice model, Clim. Dyn., 9, 71-80.

Timmermann, R., S. Danilov, J. Schröter, C. Böning, D. Sidorenko, and K. Rollenhagen (2009), Ocean circulation and sea ice distribution in a finite element global sea ice ocean model, Ocean Modelling, doi:10.1016/j.ocemod.2008.10.009.

Uttal, T., J.A. Curry, M.G. McPhee, D.K. Perovich, R.E. Moritz, J.A. Maslanik, P.S. Guest, H.L. Stern, J.A. Moore, R. Turenne, A. Heiberg, M.C. Serreze, D.P. Wylie, P.O.G. Persson, C.A. Paulson, C. Halle, J.H. Morison, P.A. Wheeler, A. Makshtas, H. Welch, M.D. Shupe, J.M. Intrieri, K. Stamnes, R.W. Lindsey, R. Pinkel, W.S. Pegau, T.P. Stanton, and T.C. Grenfeld (2002), Surface heat budget of the Arctic Ocean, Bull. Am. Meteorol. Soc., 83, 255-275.

Vihma, T., J. Hartmann, and C. Lüpkes (2003), A case study of an on-ice air flow over the Arctic marginal sea ice zone, Boundary-Layer Meteorol., 10\%, 189-217.

Weiss, A.I., J. King, T. Lachlan-Cope, R. Ladkin (2011), On the effective aerodynamic and scalar roughness length of Weddell Sea ice, J. Geophys. Res., 116, D19119, doi:10.1029/2011JD015949. 
Wu, J., (1980), Wind-stress coefficients over sea surface near neutral conditions-a revisit. J. Phys. Oceanogr., 10, 727-740.

Zhang J., and D.A. Rothrock (2003), Modeling global sea ice with a thickness and enthalpy distribution model in generalized curvilinear coordinates, Mon. Wea. Rev., 131, 845861. 
Figure 1. Sea ice morphological structure in the MIZ (top, Photo: C. Lüpkes) (width: $\approx 100 \mathrm{~m}$ ) and in the central Arctic during summer (bottom, Photo: S. Hendricks) (width: $\approx 300 \mathrm{~m}$ ). Floes in the MIZ are surrounded by open water with the possibility of finding a more or less straight path across the region within water, while ponds and leads appear to be surrounded by sea ice.

Figure 2. Form drag coefficients (left vertical axis) as a function of sea ice concentration $A$ obtained with equations (8) using the $F$ function (9) (long-dashed line) and $F=0.21$ (solid line). The short-dashed line represents $F$ (right vertical axis) obtained from equation (9); the dash-dotted line shows $F=0.21$.

Figure 3. Observations (filled circles) with mean statistical error (see text) and approximations of freeboard $h_{f}$ (blue line: equation (24); red line: equation (25); black dashed line: equation (23)), floe length $D_{i}$ (red line: equation (26) with $\beta=1$; long-dashed green line: $D_{i}=25 \mathrm{~m}$, black short-dashed line: equation (4)), and aspect ratio $\alpha_{r}=D_{i} / h_{f}$ (black dashed line: equations (23) and (4); red line: equations (25) and (26) with $\beta=1$; green long dashed line: equation (25) and $\left.D_{i}=25 \mathrm{~m}\right)$.

Figure 4. Sheltering function $S_{c}$ (equation 28), with $s=0.5, D_{w}$ from equation (29), and normalized dynamical pressure on floe edges $P_{0}$ (equation 21) as a function of the sea ice concentration $A$ (for $\beta=1, c_{w}=0.3, h_{f}$ from equation (25)).

Figure 5. Sheltering function $S_{c}^{2}$ obtained with equations (28), (30), and (31) as a function of sea ice concentration $A$ for different values of $\beta$ (solid line: equation (28); short-dashed line: (30); long-dashed line: (31), $\beta$ is $0.5,1.0$, and 1.5 from left to right lines).

Figure 6. Floe distance $D_{w}$ from equation (29) (top) and number density $n$ from equation (32) with $c_{s}=1$ (bottom) as a function of sea ice concentration (thick solid lines: use of equations (26) and $A_{\star}$ from (27); dashed lines: use of (26) assuming $A_{\star}=1$; dash-dotted line: use of (4)). 
Figure 7. Drag coefficients as a function of the sea ice concentration A (black solid line: AN10, equations (1), (6), and $(35,36)$ with $\beta=1, C_{f}=2.24 \cdot 10^{-3}$; dashed lines: equation $(35,36)$ with $C_{f}=3.67 \cdot 10^{-3}$ and $\beta=1$ (green), $\beta=0.3$ (blue), and $\beta=1.4$ (red); colored solid lines: equation $(33,34)$ with $D_{\min }=8 \mathrm{~m}, D_{\max }=300 \mathrm{~m}, h_{\max }=0.55 \mathrm{~m}, h_{\min }=0.35 \mathrm{~m}, \beta=1$ (green), $\beta=1.4$ (red), $\beta=0.3$ (blue). In all cases, $C_{d, w}=1.5 \cdot 10^{-3}, C_{d, i}=1.6 \cdot 10^{-3}$, except for the black solid line, where $C_{d i}=1.4 \cdot 10^{-3}$. Symbols represent observations (MIZEX: Guest and Davidson (1987) (triangles), Anderson (1987) (asterisks); REFLEX: circles with error bars; Schröder et al. (2003) (squares); Antarctica: Andreas et al. (1984) (diamonds).

Figure 8. Number of melt ponds per square meter as calculated with equation (38) assuming quadratic (solid line) and circular (dashed line) ponds. Symbols represent NSIDC data [Fetterer et al., 2008], which were averaged in classes with $10 \%$ steps in ice concentration.

Figure 9. Left: Observations [Fetterer et al., 2008] of mean pond size (area) and parametrization assuming ponds as squares and using equation (45, solid line). Right: Observations as in the left figure but mean edge length is shown assuming a quadratic shape of melt ponds. Solid line results from equation (45) and dashed line from equation (46). Ice concentration is here the fraction of ice cover without ponds.

Figure 10. Possible approximations of the drag coefficients in the range $0.4 \leq A \leq 1$ over summer sea ice (symbols in both figures represent SHEBA measurements at different masts as analyzed by AN10). Top, green: equation (48); brown: equation (49); $\mu=\nu=1, h_{e}=1.2 \mathrm{~m}$ and $z_{0, w}=3.27 \cdot 10^{-4} \mathrm{~m}, C_{d, w}=1.5 \cdot 10^{-3}, C_{d, i}=1.5 \cdot 10^{-3}$ in both curves; red: equation $(43)$ with $h_{p}=0.25 \mathrm{~m}$ and $D_{w}^{\prime}$ from (45); blue: AN10 (equations (1) and (50)) using $C_{d, i}=1.4 \cdot 10^{-3}$ and other parameter values as above. Bottom: equation (48) with different parameter values. 
Figure 11. Drag coefficients for the MIZ and inner Arctic. Black dashed lines: parametrization with equation (22) for different $\beta$ starting with the value 1.8 for the lowermost line and decreasing in steps of 0.3 towards the uppermost line); red symbols: SHEBA observations as in Figure 10); black symbols: MIZ observations as in Figure 7; red solid line as green curve in Figure 10. 


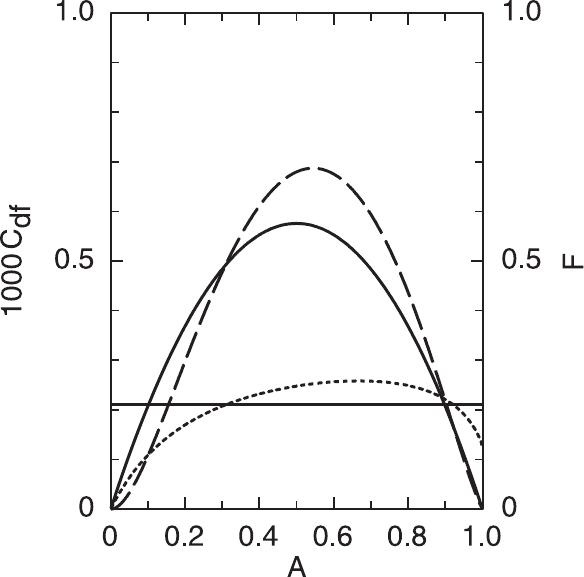



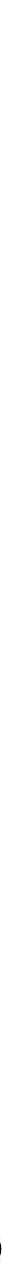


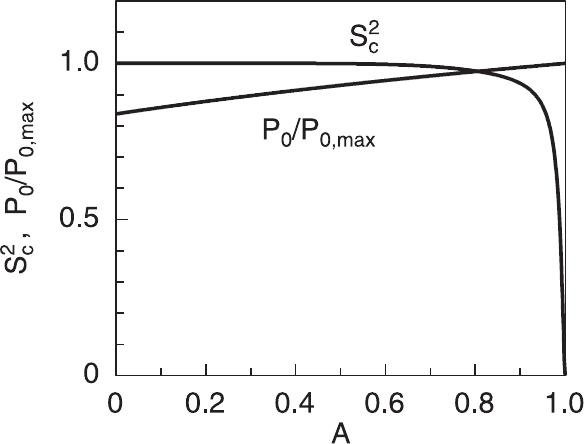


N

1.0

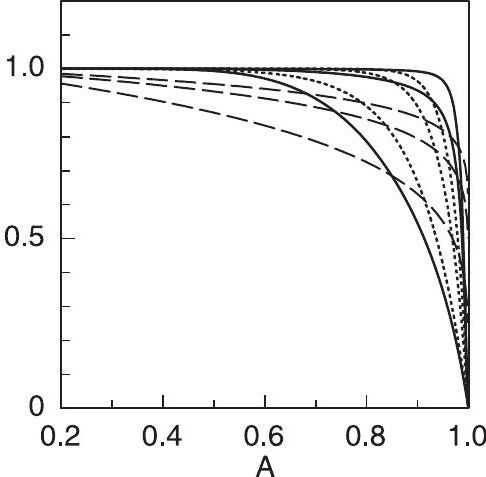

$\omega^{\circ}$ 


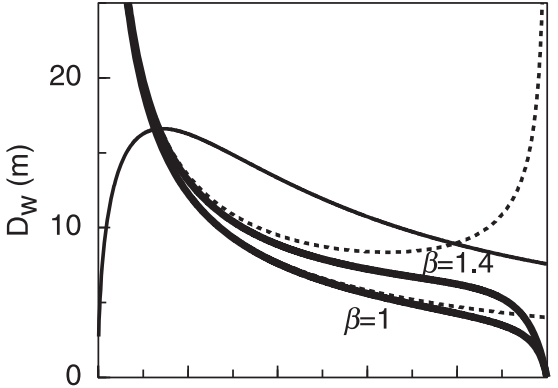

ธิ

0.003

0.002

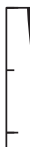

0.001 


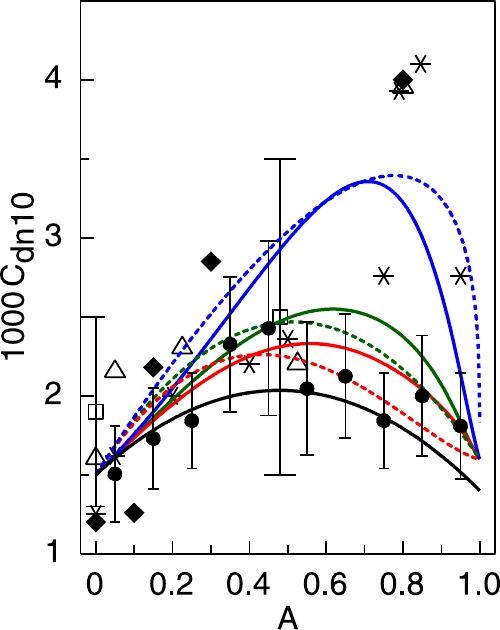




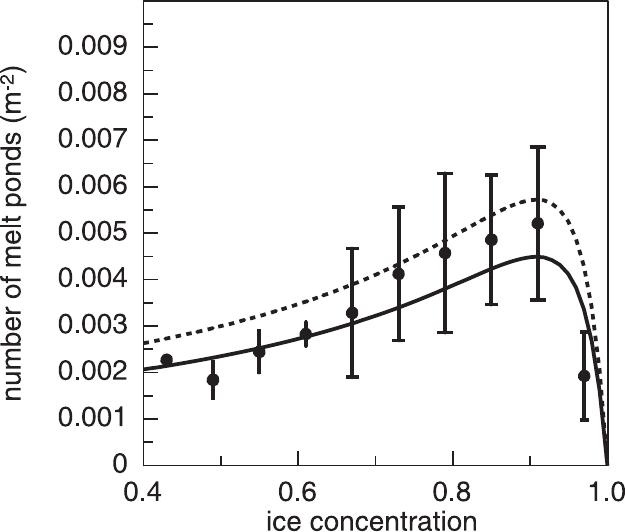



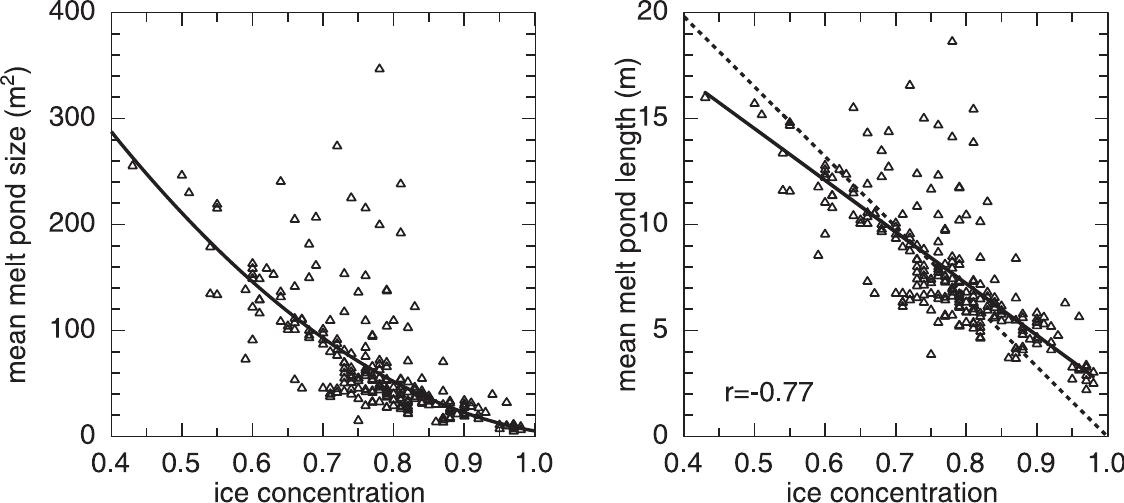
ice concentration 

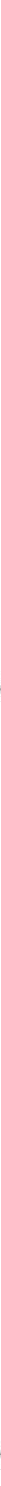


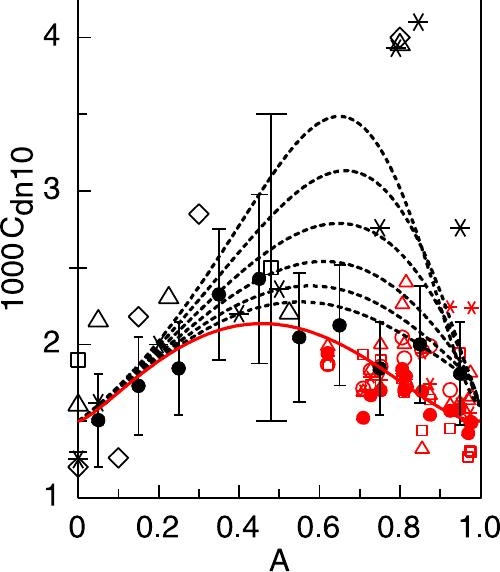

